\title{
Halogen etching of Si via atomic-scale processes
}

\author{
C.M. Aldao ${ }^{\text {a,* }}$, J.H. Weaver ${ }^{b}$ \\ a Institute of Materials Science and Technology (INTEMA), \\ Universidad Nacional de Mar del Plata-CONICET, Juan B. Justo 4302, 7600 Mar del Plata, Argentina \\ ${ }^{\mathrm{b}}$ Department of Materials Science and Engineering, University of Illinois at Urbana-Champaign, \\ 1304 West Green Street, Urbana, 61801 IL, USA
}

\begin{abstract}
Scanning tunneling microscopy (STM) studies of spontaneous halogen etching of $\operatorname{Si}(100)$ $2 \times 1$ and $\mathrm{Si}(111)$ in the range 700-1100 K are reviewed. Although the morphology depends on temperature, the steady-state removal of $\mathrm{Si}$ by chlorine, bromine and iodine is dominated by layer-by-layer etching that produces bounded surface roughness. For $\operatorname{Si}(100)$, the etch pits, step profiles, and Si regrowth structures on the exposed surfaces exhibit temperaturedependent characteristic patterns. Healing of this etched surface begins at $\sim 1000 \mathrm{~K}$, and there is complete halogen desorption and restoration of the pre-etch morphology by $\sim 1100$ $\mathrm{K}$. Since reaction pathways involve atomic level interactions, it is possible to use the data obtained with STM to extract information about the atomic-scale processes involved during etching. Thermally activated reactions of adsorbed $F$ show that dimer vacancies (DVs) are produced in the top layer but, more significantly, there is multilayer pitting that accounts for a surface roughening which is unique to $\mathrm{F}$. For $\operatorname{Si}(111)-7 \times 7$ etching in the range $700 \leqslant T \leqslant 900 \mathrm{~K}$ involves Si removal from adatom sites and conversion to a $1 \times 1$ periodicity that is stabilized by the halogen. In this temperature range, bilayer step flow etching dominates and regrowth structures derived from six-membered Si rings terminated by $\mathrm{Br}$ appear near the bilayer steps. Step flow continues at $1000 \mathrm{~K}$ but terrace pitting is also activated. This produces triangular bilayer pits bounded by $\langle 1 \overline{1} 0\rangle$ edges. At $1100 \mathrm{~K}$, etching produces disordered vacancy clusters in the adatom layer. The presence of small ordered domains amidst randomly distributed adatoms is attributed to facile local removal. (C) 2001 Elsevier Science Ltd. All rights reserved.
\end{abstract}

Keywords: Scanning tunneling microscopy; Etching; Surface structure, morphology, roughness and topography; Silicon; Halogens

\footnotetext{
${ }^{*}$ Corresponding author. Tel.: +54-223-481-6600; fax: +54-223-481-0046.

E-mail address: cmaldao@mdp.edu.ar (C.M. Aldao).
} 


\section{Acronyms}

LD line defects

ML monolayer

STM scanning tunneling microscopy

TPD temperature-programmed-desorption

\section{Contents}

1. Introduction . . . . . . . . . . . . . . . . . . . . . . . . . . 191

2. Silicon surfaces . . . . . . . . . . . . . . . . . . . . . . . . . . . . 192

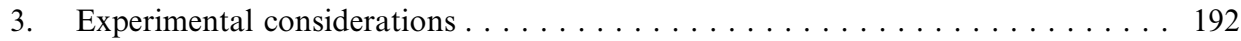

4. Halogen adsorption on $\operatorname{Si}(100)-2 \times 1$ and etching $\ldots \ldots \ldots \ldots \ldots$

5. $\operatorname{Si}\left(\begin{array}{lll}0 & 0\end{array}\right)-2 \times 1$ steady-state etching by bromine $\ldots \ldots \ldots \ldots \ldots \ldots \ldots$

6. Pattern analysis . . . . . . . . . . . . . . . . . . . . . . . . . . . 199

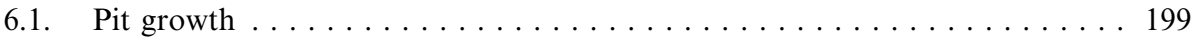

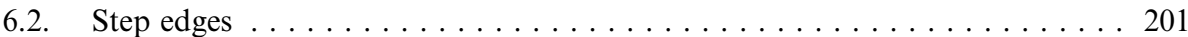

6.3. Dimer chain . . . . . . . . . . . . . . . . . . . . . . . . . 202

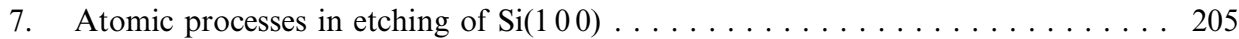

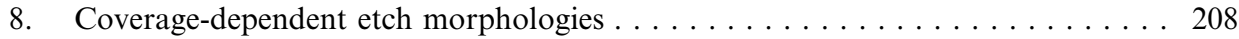

9. Vacancy creation and etching pathway $\ldots \ldots \ldots \ldots \ldots \ldots \ldots \ldots \ldots \ldots$

10. Consequences of $3 \times 1$ reconstruction $\ldots \ldots \ldots \ldots \ldots \ldots \ldots \ldots \ldots \ldots \ldots$

11. Three-dimensional pitting in etching $\operatorname{Si}(100)-2 \times 1$ with $\mathrm{F} \ldots \ldots \ldots \ldots \ldots$

12. $\quad \operatorname{Si}(111)$ etching . . . . . . . . . . . . . . . . . . . . . . . . 219

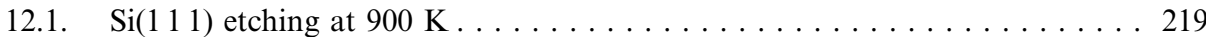

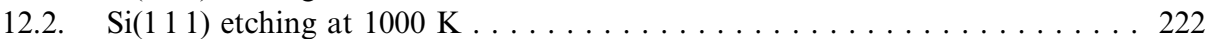

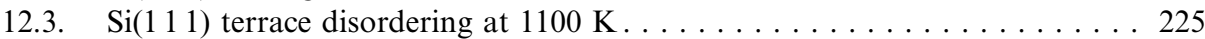

13. Conclusions. . . . . . . . . . . . . . . . . . . . . . . . . . . . . . . . 227

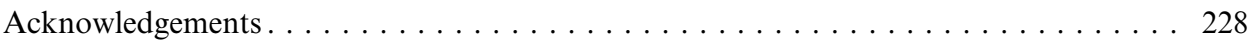

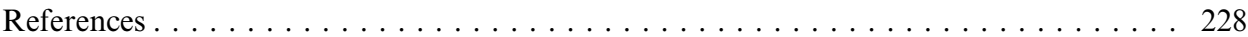




\section{Introduction}

The removal of material from surfaces by etching is crucial in many industries. For example, the microelectronics industry relies on the formation of precise geometrical structures that are produced by patterning in silicon. In wet etching, the etchant molecules react with atoms of the exposed surfaces to form reaction products that dissolve, thereby removing material. In dry etching, the surface is exposed to gaseous molecules and the etch products evaporate into the gas phase. Wet etching is isotropic, occurring both parallel and normal to the plane of the substrate. Dry etching is mostly unidirectional, and this allows more precise definition of the boundaries of the etched region [1,2].

Although chemical etching has been practiced for centuries, the detailed atomicscale mechanisms are only now being revealed. Recent insights into the etching of semiconductor surfaces by halogen atoms have come from studies that probe surface structures with atom scale precision using scanning tunneling microscopy (STM) [3]. These investigations complement studies that have explored halogen adsorption, surface chemistry, and nature of the species liberated from the surface [4-7]. The latter have established the etch products and overall etch rates, and STM has brought atomic level insight into structural changes that accompany spontaneous etching [8-15]. To date, STM has allowed us to visualize post-etching morphologies for model systems and, from the evolving surface morphology, to gain a deeper understanding of the etch processes. The ultimate goal is to combine all these results to formulate a comprehensive atomic picture of semiconductor etching.

This review discusses the etching by $\mathrm{I}, \mathrm{Br}, \mathrm{Cl}$, and $\mathrm{F}$ of $\mathrm{Si}(100)-2 \times 1$ and by $\mathrm{Br}$ of $\operatorname{Si}\left(\begin{array}{lll}1 & 1 & 1\end{array}\right)-7 \times 7$. A priori, one might think that etching phenomena might be too complicated for simple analysis, or the involved energies for the different processes would be so similar that the etched surface would become too rough for a probe like STM to be able to distinguish order. From an analysis of post-etch STM images, we found that this is not the case. Instead, the surfaces obtained after etching with $\mathrm{Cl}, \mathrm{Br}$, and I exhibit definite patterns in $\operatorname{Si}(100)$. By investigating rates of different processes, we are then asking how much one pathway is favored over another. Interestingly, the kinetic differences are sufficient to provide a controlled evolution of the surface morphology. Terrace pitting disrupts the local surface bonding and leaves the surfaces atoms in a variety of different bonding sites, each with its own barrier for desorption. Our picture of etching controlled by desorption barriers assumes that halogens can discriminate among different sites. This need not be the case. Fluorine can insert directly into bulk $\mathrm{Si}-\mathrm{Si}$ bonds, even at room temperature. Such insertion is unlikely for $\mathrm{Cl}, \mathrm{Br}$, and $\mathrm{I}$, however, and our desorption barrier picture is applicable. In much of the following discussion, we focus on $\mathrm{Br}$ etching, because it is a representative of these less reactive halogens.

The bonding configurations of $\operatorname{Si}(111)-7 \times 7$ are more varied than those of $\operatorname{Si}\left(\begin{array}{lll}1 & 0\end{array}\right)$. Etching of this surface with oxygen [16,17] has been characterized by 
material removal from steps and the formation of bilayer pits on terraces. STM studies of wet-etched $\mathrm{Si}(111)$ [18-20] showed directional anisotropies as well as terrace pitting and step retreat. STM investigations of the interaction of beams of $\mathrm{Cl}_{2}$ with $\operatorname{Si}(111)-7 \times 7$ [21-24] have emphasized chemisorption dynamics leading to preferred bonding sites and the initial formation of volatile species. Here, we present STM studies of their interaction of $\mathrm{Br}$ with $\mathrm{Si}(1111)-7 \times 7$. Temperature-, flux-, and fluence-dependent studies indicate etching from the $\mathrm{Si}$ adatom layer and transformation from the $7 \times 7$ reconstruction into a Br-stabilized $1 \times 1$ structure. We also found step retreat characterized by directional anisotropies, regrowth structures involving six-membered $\mathrm{Si}$ rings, and terrace roughening due to flux-dependent pitting.

\section{Silicon surfaces}

The geometric arrangement of atoms at surfaces can differ from what might be expected based on the bulk structure of the crystal. Surface atoms that rearrange to minimize the surface result in a reconstructed surface. In semiconductors, rear-

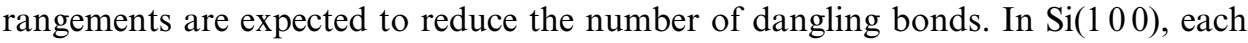
exposed atom would have two dangling bonds if bulk terminated, but small nuclear shifts allow dimerization as two of the dangling bonds convert into a surface dimer $\sigma$-bond and a $\pi$-bond forms [25].

The $\operatorname{Si}\left(\begin{array}{lll}1 & 1 & 1\end{array}\right)$ surface rearrangement is much more complicated. Indeed, the lattice reconstruction involves several layers and adopts a $7 \times 7$ reconstruction [26]. The unit cell of the reconstructed surface presents 19 dangling bonds compared to the 49 bonds corresponding to a bulk-terminated surface. The $7 \times 7$ reconstruction extends beneath the surface to include a second Si layer called the rest-layer. Here, triangular-shaped arrays of silicon atoms are arranged locally in the usual Si bulk structure. Each array is separated from the next by a bordering row of silicon dimers, similar to those found on the (1 $\left.\begin{array}{lll}0 & 0\end{array}\right)$ surface. However, on the (lllll 111$)$ surface, dimers introduce a subtle shift in the registry, known as a stacking fault. These dimer rows also intersect at the corners of each $7 \times 7$ cell, producing holes that extend through the top surface layer.

These differences in reconstruction for (1 $\left.\begin{array}{lll}1 & 0\end{array}\right)$ and (1 1111$)$ surfaces have profound consequences in terms of etching. For $\mathrm{Si}\left(\begin{array}{lll}1 & 0 & 0\end{array}\right)$, silicon atoms spontaneously dimerize when they are exposed by removal of the layer above them. For $\operatorname{Si}\left(\begin{array}{ll}1 & 1\end{array}\right)$, halogen atoms stabilize the exposed bulk structure. In this case, halogen insertion into strong $\mathrm{Si}-\mathrm{Si}$ bonds is needed for the reacted product to form and etching is expected to be slowed.

\section{Experimental considerations}

Two different kinds of experiments will be considered here. One examined morphologies produced under steady-state conditions at low flux. The term steady-state 
is used here to denote an average surface structure that does not change with exposure. Though a particular feature will change its appearance, statistical populations and ensemble characteristics are not modified. Thus, steady-state morphologies represent a balance between etching, regrowth, and diffusion effects involving pits, islands, and step edges. These experiments were conducted using a Park Scientific Instruments STM in a vacuum chamber having a base pressure of $5 \times 10^{-11}$ Torr. The other involved exposure to halogens at ambient temperature and then heating to resent temperatures for fixed amounts of time (as in temperature programmed desorption (TPD) or TPD-like experiments). These experiments were performed in a dual-chamber ultrahigh vacuum system that operates with a common base pressure of $5 \times 10^{-11}$ Torr. One chamber contained an Omicron STM1 head while the other housed the sample heating capabilities, the halogen sources, and a double-pass cylindrical mirror electron energy analyzer for surface chemical analysis.

Silicon wafers oriented within $0.2-0.5^{\circ}$ of $\left(\begin{array}{lll}1 & 0 & 0\end{array}\right)$ and miscut toward [ $\left.\begin{array}{llll}0 & 1 & 1\end{array}\right]$ were degassed at $875 \mathrm{~K}$ for several hours and then flashed to $1476 \mathrm{~K}$ for $1-2 \mathrm{~min}$. This produced well-ordered $\mathrm{Si}\left(\begin{array}{lll}1 & 1 & 1\end{array}\right)-2 \times 1$ surfaces. Analysis of clean surface images indicated a defect density of $\sim 1.3 \times 10^{13} \mathrm{~cm}^{-2}$, primarily in the form of missing di-

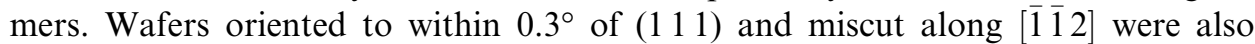
degassed at $875 \mathrm{~K}$ and flashed to $1475 \mathrm{~K}$ to obtain well-ordered $\mathrm{Si}(100)-7 \times 7$ with defect densities $\leqslant 1 \%$. Sample temperatures were monitored with an optical pyrometer and were accurate to $\pm 20 \mathrm{~K}$.

Electrochemically etched tungsten tips were cleaned using electron bombardment. Scan dimensions were calibrated with the lattice constant of the $\operatorname{Si}(100)-2 \times 1$ surface and the height of monatomic steps, and against the size of the $\operatorname{Si}(111)-7 \times 7$ unit cell and the height of bilayer steps. Electrochemical cells were used as halogen sources. Under an applied voltage, halogen ions diffuse to a Pt mesh electrode where they desorbed as dihalides. The sample was placed $\sim 3 \mathrm{~cm}$ from the mesh during exposures and then loaded onto the STM stage in a different part of the chamber. The chamber pressure remained below $1 \times 10^{-10}$ Torr during dosing.

In the steady-state experiments, exposures were done under conditions of constant flux for predetermined amounts of time so that they are quoted as a fluence in units of $\mathrm{mA}$ s. Stable cell currents of $2-120 \mu \mathrm{A}$ were used, releasing $\sim 6.25 \times 10^{12}$ to $\sim 3.75 \times 10^{14}$ molecules per second as calibrated using Faraday's law. The sample was cooled rapidly to room temperature immediately upon terminating the flux.

In the TPD-like experiments, the halogen uptake was determined by counting individual adsorption sites using STM images acquired after exposures to a given fluence at room temperature. For $\mathrm{Br}$, the surface concentration, $\theta$, increased to 0.30 ML after $15 \mathrm{~s}$, to $0.95 \mathrm{ML}$ after $300 \mathrm{~s}$, and it saturated by $600 \mathrm{~s}$ with one $\mathrm{Br}$ atom per Si surface atom $\left[1 \mathrm{ML}=6.8 \times 10^{14}\right.$ at $\mathrm{cm}^{-2}$, the planar density of $\left.\mathrm{Si}(100)\right]$. The uptake of $\mathrm{Cl}$ was somewhat more efficient, suggesting a higher sticking coefficient. From the STM images, we found no evidence of a disordered reaction layer that would indicate $\mathrm{Br}$ or $\mathrm{Cl}$ penetration beneath the surface, as is found for $\mathrm{O}$ [27] and $\mathrm{F}$ [2].

In the TPD-like studies, the samples were heated to promote thermal etching after the initial coverages were established. Typically, the temperature was raised at a rate 
of $\sim 2.8 \mathrm{~K} \mathrm{~s}^{-1}$, held at $800 \mathrm{~K}$ for $10 \mathrm{~min}$, and then decreased rapidly to room temperature. This time-temperature regimen was chosen because it reduced the halogen concentration to $50-80 \%$ of the starting concentration, as determined by counting the adsorption features using the STM.

\section{Halogen adsorption on $\mathrm{Si}(100)-2 \times 1$ and etching}

Clean surfaces are the ideal starting point in an experiment that is intended to determine atomic-scale processes. However, even clean surfaces have steps because surfaces are never atomically flat over large dimensions and there will be some number of defects in the terraces. Atoms in terraces, at steps and adjacent to defects all have different energy barriers associated with desorption, as we will discuss.

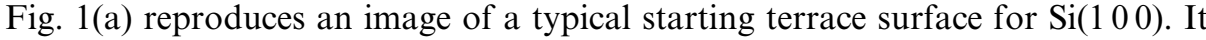
was taken with current flowing from the sample to the tip (filled-state image). The dimer row direction is along the diagonal, and dimers appear as bright protrusions centered on the rows under these biased conditions. Terrace dimers undergo dynamic buckling at $300 \mathrm{~K}$, and STM images represent a time-average charge distribution. Dimer vacancies (DVs), and steps tend to quench the buckling motion of nearby dimers. The resulting asymmetric dimers appear as bright protrusions centered on alternating atoms along the rows, labeled as ZZ. Also present are defects that appear in filled-state images as two gray protrusions on the same side of adjacent dimers. On our typical starting surface, the concentration of DVs and these C-type defects was $\sim 0.04 \mathrm{ML}$.

Diatomic molecules frequently dissociate upon chemisorption on the $\operatorname{Si}\left(\begin{array}{lll}1 & 0 & 0\end{array}\right)$ $2 \times 1$ surface. This is the case for halogens. At low coverage, single halogen atoms tend to appear on the same side of adjacent dimers in the same row at room temperature. Mild annealing at $\sim 500 \mathrm{~K}$ allows the halogen atoms to diffuse and they appear in pairs bonded to single dimers [28,29]. This is expected since bonding to both dangling bonds of a single dimer is energetically favored over bonding to single bonds of two dimers. The latter breaks two $\pi$-bonds while the former costs only one. This thermodynamic preference for $\mathrm{Cl}$ to be adsorbed on the same dimer has been demonstrated through annealing experiments in which only configurations of $\mathrm{Cl}$ saturated dimers were observed. Yates and co-workers [30] concluded that the distribution of $\mathrm{Cl}$ after room temperature adsorption is controlled by kinetic rather than thermodynamic factors.

Fig. 1(b) shows an occupied state image for a surface with a $\mathrm{Br}$ concentration of $0.88 \mathrm{ML}$. For a sample bias of $-2.5 \mathrm{~V}$, the STM tip probed states formed by $\mathrm{Br} 4 \mathrm{p}$ and $\mathrm{Si}$ dangling bonds. Dimers with adsorbed $\mathrm{Br}$ appeared brighter than bare $\mathrm{Si}$ dimers, labeled as BD. Adsorption eliminated dimer buckling, and all appeared symmetric. At this concentration, adsorption favored pair-wise occupancy of the dimers, $2 \mathrm{SiBr}$, with two distinct features. When halogen uptake was done at room temperature, we also observed dimers with only one $\mathrm{Br}$ adatom, labeled $\mathrm{SO}$ for singly occupied or 2SO if they appeared on adjacent dimers of a row. These features disappeared when diffusion was promoted, and it can be concluded that they were 

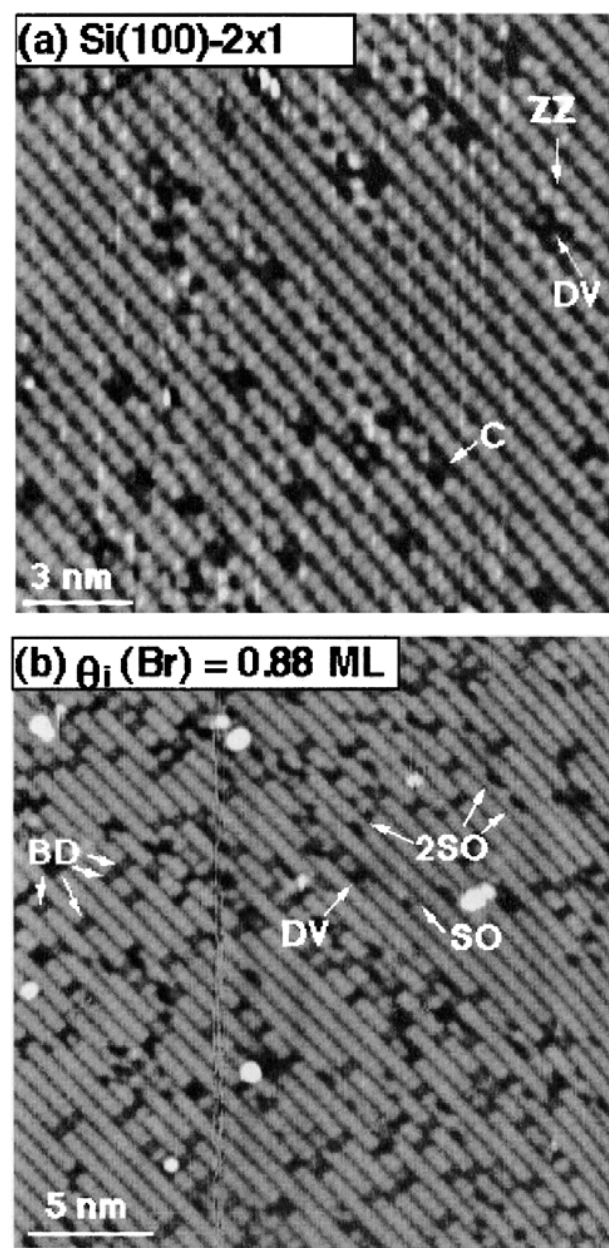

Fig. 1. (a) STM image of clean $\operatorname{Si}\left(\begin{array}{lll}1 & 0 & 0\end{array}\right)-2 \times 1$ showing dimers vacancies, labeled DV, C-type defects, C, and rows of asymmetric buckled dimers near defects, ZZ. Sample bias $-2.0 \mathrm{~V}$ (to probe the occupied states); tunneling current $0.2 \mathrm{nA}$. (b) STM image of $\mathrm{Si}(100)-2 \times 1$ after exposure to $\mathrm{Br}_{2}$ at room temperature where the $\mathrm{Br}$ coverage, $\theta(\mathrm{Br})$, was $0.88 \mathrm{ML}$. The darkest features are $\mathrm{DVs}$; bare $\mathrm{Si}$ dimers, BD, appear with intermediate gray scale; and dimers with two $\mathrm{Br}$ adatoms, $2 \mathrm{SiBr}$, are the dominant species. $\mathrm{SO}$ denotes a dimer with a single $\mathrm{Br}$ adatom. These features often appear in pairs on the same row but they disappear after mild annealing because $\mathrm{Br}$ can access the lower-energy state 2 $\mathrm{SiBr}$ (a dimer with one $\mathrm{Br}$ bonded to each dangling bonds). Sample bias $-2.5 \mathrm{~V}$; current $0.2 \mathrm{nA}$. The images were acquired at room temperature.

converted to pairwise-occupied dimers as discussed above. The brightest spots in Fig. 1(b) were probably weakly bonded terrace $\mathrm{SiBr}_{x}$ moeities, presumably formed as a consequence of the highly exothermic uptake of the halogens. Indeed, comparison of the starting surface and the halogen-exposed surface indicated an increase in a number of vacancies [11]. 


\section{5. $\operatorname{Si}(100)-2 \times 1$ steady-state etching by bromine}

Studies of the etch patterns and their formation, growth, and evolution under steady-state conditions make it possible to measure differences in the effective energy barriers for the different processes that take place during etching. Once a single vacancy is created on a terrace, it destabilizes its neighbours and vacancy growth will produce a linear vacancy or a branched vacancy. For a pit of, say seven vacancies in a dimer row, growth will result in either an eight-unit line or a branched structure. Quantitative analysis of STM images indicates that removal events favor linear growth over branching, indicating an energy difference of only $0.14 \mathrm{eV}$. Although energy differences of this amount are small compared to a desorption energy of about $3 \mathrm{eV}$, they are sufficient to account for the patterns observed during etching.

Figs. 2(a)-(c) display constant-current occupied-state images from surfaces that had reached steady-state conditions for continuous etching at 700,800 , and $900 \mathrm{~K}$. Inspection reveals that the specific morphologies depend on temperature but also that there are common temperature-independent structures [10]. The terraces show dark areas which are elongated along the dimer row direction that are single-layer deep etch pits consisting missing dimer rows. Also present are extended vacancy complexes. Bright lines that run perpendicular to these pits are $\mathrm{Si}$ dimer chains that have grown on the terrace. These features are formed from Si atoms released during etching from terrace and steps sites. Some of these atoms can diffuse on the surface forming stable nuclei that grow on the terraces.

In general, the etch pits and terrace chains are only a few dimer-units in size on surfaces etched at $700 \mathrm{~K}$ or lower (Fig. 2(a)). They exhibit short $2 \times 1$ chains and small, single-layer etch pits that are distributed evenly on the terraces. High resolution scans show that nearly all Si dangling bonds are saturated by $\mathrm{Br}$ atoms, including those within pits and on chains, and that the $2 \times 1$ reconstruction is maintained. The density of pits and chains is low and the chains remain isolated, i.e., features wider than two rows were not observed. Comparison of the step roughness after etching to that for the starting surface demonstrates that the roughness increases. Etching at $700 \mathrm{~K}$ then occurs primarily at step edges, supplemented by limited etching at native terrace defects where $\mathrm{Si}-\mathrm{Si}$ bonds may be more easily broken than on pristine terraces.

Etching at $800 \mathrm{~K}$ results in a dramatic change in the steady-state morphology, Fig. 2(b), with more local height variation because etch pits and islands constitute $\sim 30 \%$ of the area of terraces. At $800 \mathrm{~K}$, these features are larger and exhibit increased shape anisotropies. An inverse in step roughness at $800 \mathrm{~K}$ is apparent by comparing to Fig. 2(a). Increased roughness results from a combination of thermally enhanced etching into the terrace and the regrowth of free Si. Hence, etching at 800 $\mathrm{K}$ can be characterized as the creation and growth of single-layer etch pits on terraces as a complement to step etching. Islands remain two-dimensional rather than evolving into rough, three-dimensional structures, and no more than three layers are exposed on a given terrace.

Fig. 2(c) shows a terrace after etching at $900 \mathrm{~K}$. Here, two domains of $2 \times 1$ and $3 \times 1$ character are present. High resolution images show that the $3 \times 1$ domains are 

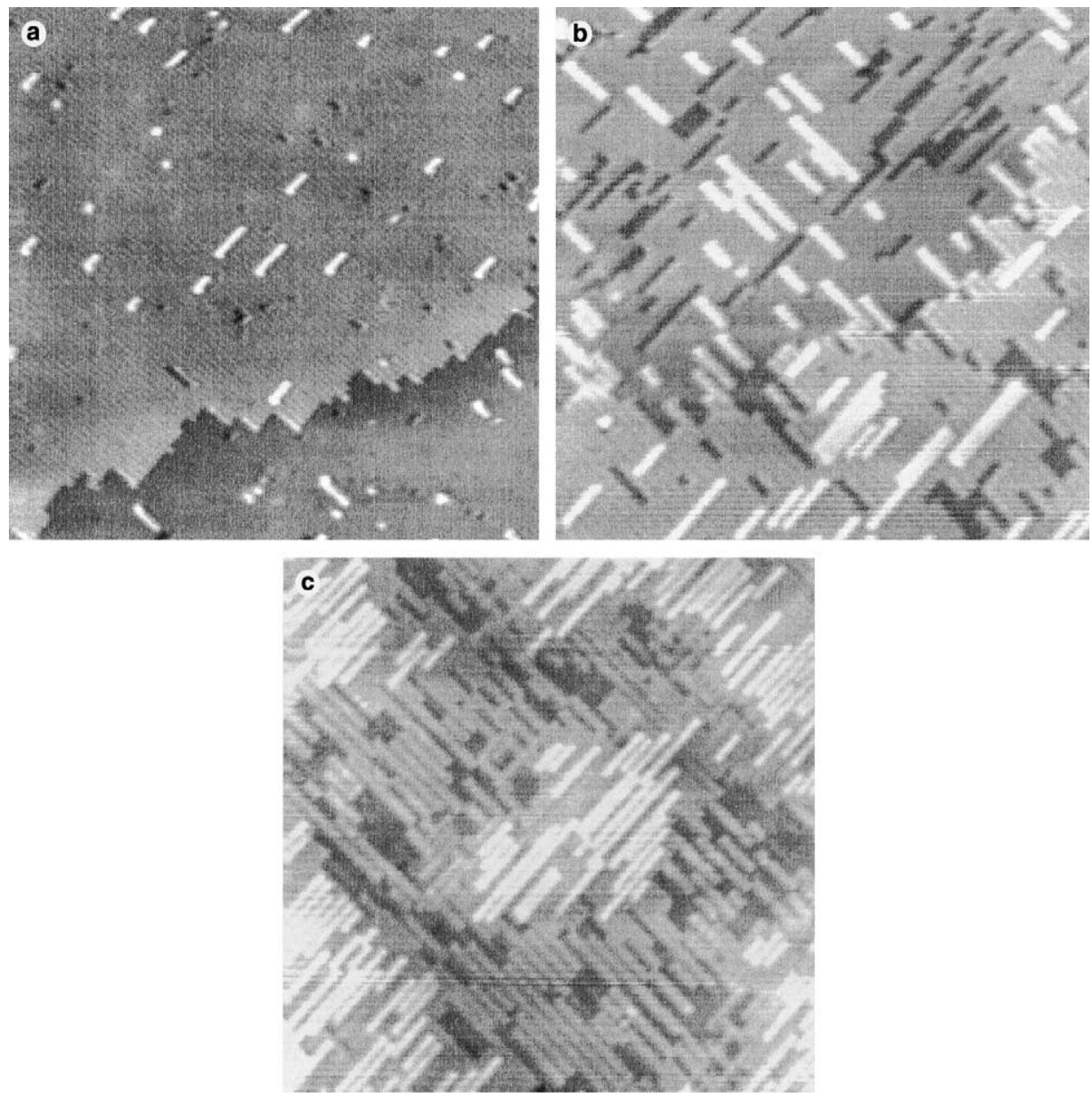

Fig. 2. STM images of $\mathrm{Si}(100)-2 \times 1$ exposed to $\mathrm{Br}_{2}$ showing changes in steady-state morphologies for etching at 700-900 K. The etch pits and regrowth chains increase in number and size with increased temperature. An $S_{\mathrm{B}}$ step crosses image (a) and another is evident in (b). Etching at $700 \mathrm{~K}$ is limited by the rate of $\mathrm{SiBr}_{2}$ desorption and it occurs largely at steps. Terraces remain relatively defect-free and $\mathrm{Br}$-saturated at $700 \mathrm{~K}$, but enhanced terrace etching is evident at $800 \mathrm{~K}$ where remnants of the terrace resemble regrowth structures. The morphology achieved by etching at $900 \mathrm{~K}$ reflects the formation of a $3 \times 1$ phase derived from rows of $\mathrm{SiBr}_{2}$ separated by $\mathrm{SiBr}$ dimer rows. As depicted in Fig. 13, $\mathrm{SiBr}_{2}$ desorption results in missing atoms rows.

composed of $\mathrm{Si}$ dimer rows separated by missing atom rows [8]. Thus, $3 \times 1$ patterning requires a sufficient local concentration of $\mathrm{Br}$ and temperature in the range $\sim 850-900 \mathrm{~K}$. The conversion of Br-terminated $2 \times 1$ domains to $3 \times 1$ domains requires the coordinated breaking of dimer bonds with redimerization that produces $\mathrm{SiBr}_{2}$ rows $\mathrm{Br}$-terminated $\mathrm{Si}$ dimer rows. The volatile $\mathrm{SiBr}_{2}$ rows are unstable against desorption and their departure leaves rows of Br-terminated dimers separated by missing atom rows. Their absence as extended domains in these cases emphasizes the 
restricted conditions under which patterning occurs. While some $2 \times 1$ islands due to standard regrowth processes are found after etching at $900 \mathrm{~K}$, it is clear that the dominant $3 \times 1$ morphology results from this specific mechanism.

Exposure of $\mathrm{Si}(100)-2 \times 1$ to $\mathrm{Br}_{2}$ at 1000 and $1100 \mathrm{~K}$ results in steady-state etching that is characterized by scattered small pits and patches of clean Si among Br-terminated areas, as shown in Figs. 3(a) and (b). Images obtained after etching at
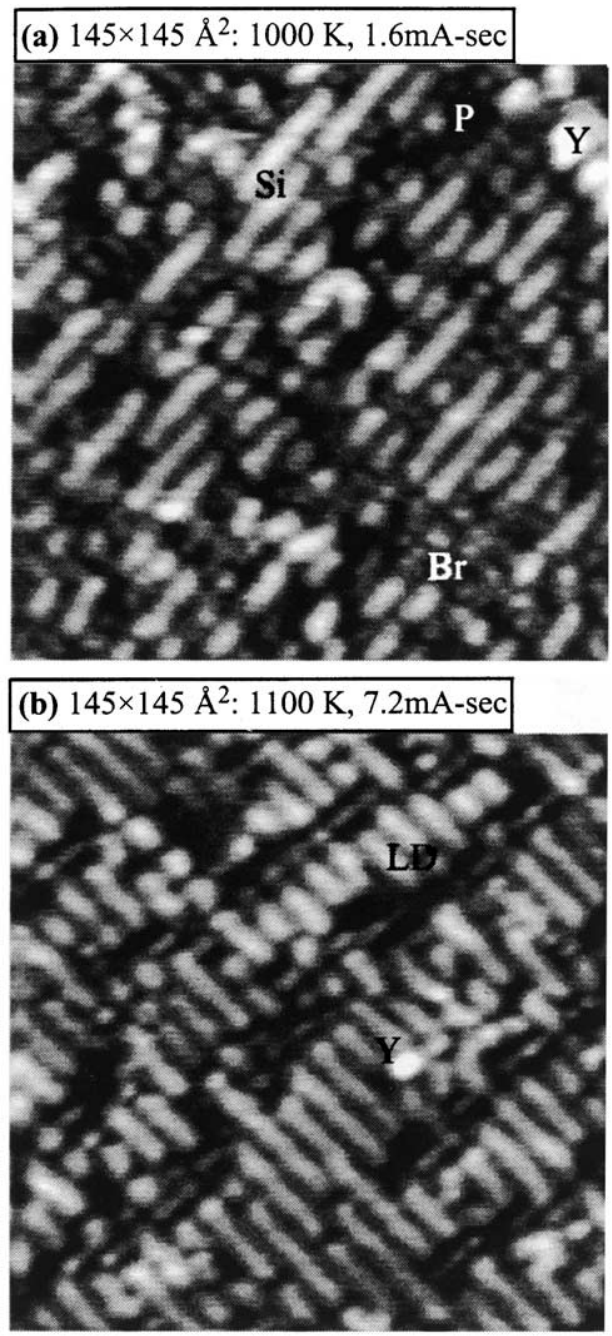

Fig. 3. Small-scale images taken after steady-state etching at 1000 and $1100 \mathrm{~K}$. Bromine-free Si dimers, labeled $\mathrm{Si}$, are evident in (a) and $\mathrm{Br}$ adsorption sites, $\mathrm{Br}$, are mixed with irregularly shaped pits, $\mathrm{P}$. The bright feature labeled Y represents a disordered regrowth structure. (b) DVs created randomly on terraces are mobile at $1100 \mathrm{~K}$ and they interact to form $\mathrm{LD}$ that are elongated perpendicular to the dimer row direction. The surface concentration of $\mathrm{Br}$ is low during etching at $1100 \mathrm{~K}$, and the etch rate is flux-limited. 
$1000 \mathrm{~K}$ show that approximately half of the surface is Br-free; there is almost no residual $\mathrm{Br}$ after etching at $1100 \mathrm{~K}$. This is consistent with TPD data that reveal complete desorption after heating bromine-exposed surfaces to $1100 \mathrm{~K}$ [30].

Etching at $1000 \mathrm{~K}$ can be characterized by a process where weakened Si-Si bonds and enhanced $\mathrm{Br}$ diffusion combine to facilitate pitting of the terraces. These defects can rapidly expand by etching at their boundaries, ultimately coalescing to create large pits. These processes expose several layers, the number of which depends on the kinetics and the terrace width. In this regime, the surface concentration limits the overall rate, and etching would increase with increased flux. This is in contrast to the behavior at lower temperature where the surface was saturated and desorption was the rate limiting factor.

Etching at $1100 \mathrm{~K}$ results in a distinct morphology because of increased reactivity, lower residence time of $\mathrm{Br}$ on the surface, and higher vacancy mobility. DVs in adjacent rows experience an attractive interaction [31-34] and LD form perpendicular to the dimer rows, Fig. 3(b). The nucleation of a line defect requires a minimum vacancy density and sufficient time and temperature for them to organize. Thus, the average defect length increases with continued exposure at $1100 \mathrm{~K}$ and the average spacing decreases so that the surface evolves into a steady-state $2 \times n$ network of LD, where $6 \leqslant n \leqslant 12$. The LD are then $25-50 \AA$ apart and individual defects can reach lengths of $\sim 30$ dimer rows.

Etching at $1000 \mathrm{~K}$ produces a large number of vacancies but these vacancies do not assemble into line defects (LD). This suggests that vacancy diffusion is reduced. Images were obtained after annealing surfaces that had been etched at $1000 \mathrm{~K}$ but without an incident flux to replenish the $\mathrm{Br}$ loss in etching. Interestingly, LD did form in this Br-depletion/anneal experiment indicating that the presence of $\mathrm{Br}$ strongly limits vacancy diffusion. Eventually, once the $\mathrm{Br}$ is completely desorbed, there is sufficient Si mobility at $1000 \mathrm{~K}$ that the etch damage heals and the surface returns to a regularly stepped morphology.

\section{Pattern analysis}

\subsection{Pit growth}

We will now focus on the atomic sequence of events that leads to etching in $\mathrm{Si}\left(\begin{array}{ll}1 & 0\end{array}\right)$ so as to determine the relative energies that give rise to the various etch patterns. Spontaneous etching can be described in terms of statistical arrival, dissociation, thermalization, and subsequent diffusion of halogens on the surface, with the ultimate desorption of volatile molecules. Surface diffusion barriers are typically much smaller than desorption activation energies, so the rate limiting process for etching is the desorption, not the transport of etchant atoms to pit edges. Pit growth, then, can be understood as a function of desorption activation energies that reflect an interactions between neighboring atoms. From an analysis of post-etch STM images, we found that surfaces exhibit definite patterns that allow insight into mechanisms of pit formation and growth [35]. 
We could model etching based on site configuration-dependent activation energies. In particular, it was shown that areal distributions of linear pits on the $\operatorname{Si}\left(\begin{array}{lll}1 & 0 & 0\end{array}\right)$ surface could be described in terms of interaction energies between first and second neighboring dimers. Etch pit initiation involves removal of a single dimer from a terrace. This DV then destabilizes adjacent dimers to a sufficient extent that the halogens can attack them and the pit grows.

In the early stages of etching, there is a dominance of linear pits that are one row wide. The fact that they are linear indicates that they are produced by addition of vacancies along the dimer row direction. In fact, there are two possibilities for pit growth, namely linear growth via dimer removal along the row direction and branch formation via dimer removal from an adjacent row. Growth of a single vacancy, $V$, along a dimer row produces a double linear vacancy, $V_{2}$. This unit can continue with linear growth to form $V_{3}$ or a dimer can be removed from the adjacent row to produce a branch. The observed densities of pits with distinct sizes and shapes then result from competition between linear and branch growth. While it is reasonable to expect that pits will follow the most probable distribution, there are complications that would randomize the distribution, such as limited availability of $\mathrm{Cl}$ and interaction between pits. The test, then, lies in analysis of the STM results.

If we assume that quasi-steady-state conditions are met, then generation of pits of a specific size by growth from a smaller unit and consumption of pits of that size by growth to the next larger unit are constant, regardless of the size. In this case, the ratio between the areal densities of chains with lengths $i+1,\left[V_{i+1}\right]$, and $i,\left[V_{i}\right]$, can be written as

$$
\left[V_{i+1}\right] /\left[V_{i}\right]=1 /\left[1+2 k_{\mathrm{EB}} / k_{\mathrm{L}}+(i-2) k_{\mathrm{MB}} / k_{\mathrm{L}}\right],
$$

where the $k$ 's are generation rates. $k_{\mathrm{L}}$ accounts for linear growth at the end of the chain, $k_{\mathrm{EB}}$ accounts for branching at the end of the chain, and $k_{\mathrm{MB}}$ accounts for branching at middle sites along the chain. For a linear pit, there are two sites for linear growth and a statistical factor of two is inherent in $k_{\mathrm{L}}$. Similarly, there are two possible sites for branching at every DV. Accordingly, the statistical factor for end point branching is four and for mid-chain branching at the rest of the vacancies is $2(i-2)$. If $k_{\mathrm{MB}}$ is negligible, then the probability for linear growth is independent of $i$ as discussed in [12].

Fig. 4 shows $\ln \left(\left[V_{i}\right] /[V]\right)$ vs. $i$ for linear pits on $\operatorname{Si}\left(\begin{array}{lll}1 & 0 & 0\end{array}\right)-2 \times 1$ after etching at 850 $\mathrm{K}$ with $\mathrm{Cl}$. This distribution was obtained by direct analysis of the STM images. The straight dashed line corresponds to the situation where all branching occurs at chain ends. The concave-downward dashed line follows if branching is equally probable at all chain sites, $k_{\mathrm{MB}}=k_{\mathrm{EB}}$. The experimental results indicate that the physical situation is closer to the first extreme since allowing branching at any chain sites with the same probability would show up as a non-straight line in the plot. (Fitting the data with $k_{\mathrm{EB}}=k_{\mathrm{MB}}$ is not possible.)

The experimental results can then be fit using Eq. (1) with $k_{\mathrm{EB}} / k_{\mathrm{L}}$ and $k_{\mathrm{MB}} / k_{\mathrm{L}}$ as parameters. Fig. 4 shows the fit obtained with $k_{\mathrm{EB}} / k_{\mathrm{L}}=0.147$ and $k_{\mathrm{MB}} / k_{\mathrm{L}}=0.049$. Assuming, as in [12,35], that rate constants can be related to activation energies via Arrhenius relationships, then expressions of the form $k_{\mathrm{B}}=\sigma_{\mathrm{B}} \exp \left[-E_{\mathrm{B}} / k T\right]$ can be 


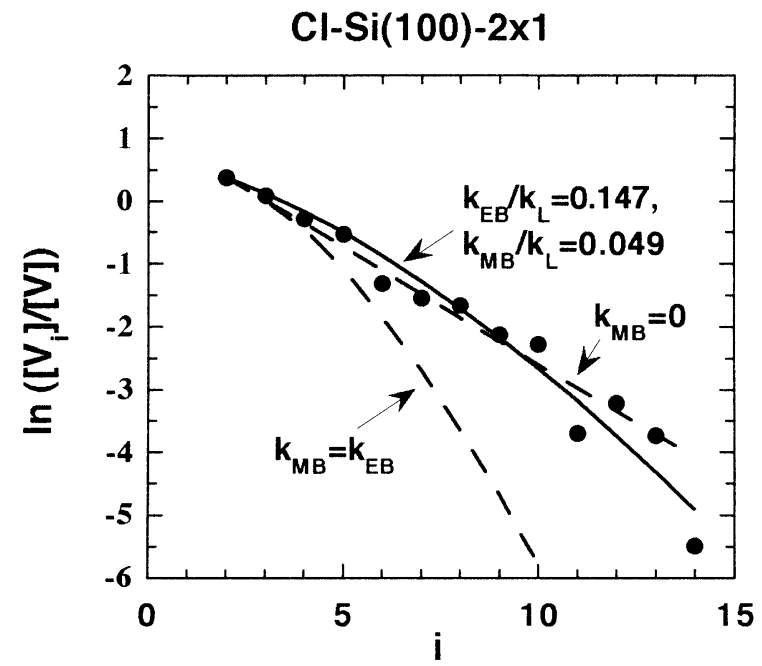

Fig. 4. Areal distribution of linear pits of size $i,\left[V_{i}\right]$, normalized to the areal density of single vacancies, [V], for $\mathrm{Si}\left(\begin{array}{lll}1 & 0 & 0\end{array}\right)-2 \times 1$ after etching at $850 \mathrm{~K}$ with $\mathrm{Cl}$. The straight line corresponds to a constant linear probability with no branching except at the end, $k_{\mathrm{MB}}=0$ (a linear pit in a dimer row grows along the dimer row without branching into a second row). The dashed line corresponds to equal chance of branching at mid-points and end points, $k_{\mathrm{MB}}=k_{\mathrm{EB}}$. The solid line represents a fit using Eq. (1) with $k_{\mathrm{EB}} / k_{\mathrm{L}}=0.147$ and $k_{\mathrm{MB}} / k_{\mathrm{L}}=0.049$.

written, from which the interaction energies could be deduced. Thus, $E_{\mathrm{EB}}-E_{\mathrm{L}}=$ $0.14 \mathrm{eV}$ and $E_{\mathrm{MB}}-E_{\mathrm{L}}=0.22 \mathrm{eV}$. Accordingly, $E_{\mathrm{MB}}-E_{\mathrm{EB}}=0.08 \mathrm{eV}$, the energy difference for dimer removal to form a branch at a mid-chain site vs. an end site. Branching occurs preferentially at chain ends with such events being three times more likely than mid-chain branching, $k_{\mathrm{EB}} / k_{\mathrm{MB}}=3$.

The removal of $\mathrm{Si}$ dimers has been simulated through a Monte Carlo scheme where events take place according to probabilities that are dictated by the dimer interaction energies. The fact that the experiments are reproduced very well indicates that the essentials of etching are included [36]. It is important to point out that in the previous analysis terraces are in quasi-steady-state conditions. While the surface does not change statistically with further etching at $700 \mathrm{~K}$, Eq. (1) is not valid because the consumption of chains of a given size is larger than generation from a smaller chain [37].

\subsection{Step edges}

The $\operatorname{Si}(100)$ surface reconstructs to form parallel rows of dimers. These dimer rows are perpendicular to each other on terraces that are separated by an odd number of monatomic steps. Adjacent steps are then inequivalent if the surface is miscut with a surface normal tilted toward [0 11 1]. Steps parallel to the dimer rows of the upper terrace, $S_{\mathrm{A}}$ steps, have few kinks and appear smooth whereas those perpendicular to the dimer rows, $S_{\mathrm{B}}$ steps, contain a high density of thermally excited 
kinks and are rougher [38,39]. Two different B-type steps can occur depending on whether the dimer row of the upper terrace ends just on a dimer row or in between two dimers rows of the lower terrace [40]. This latter configuration, the so called rebonded $S_{\mathrm{B}}$ step, is energetically favored and it is dominant since the former is characterized by extra dangling bonds. Therefore, step fluctuations are observed to occur through the attachment and detachment of sets of two dimers.

Many studies [38,39,41-49] have focused on atomic steps in $\mathrm{Si}\left(\begin{array}{lll}1 & 0 & 0\end{array}\right)$ and the equilibrium exchange of atoms between step sites and terraces. Ganz and co-workers [49] carried out high temperature STM studies that permitted a determination of the rates of attachment and detachment events involving double dimers at specific sites at $\operatorname{Si}\left(\begin{array}{lll}1 & 0 & 0\end{array}\right) S_{\mathrm{B}}$ steps. Aldao and co-workers [50] then examined the atom dynamics involved in equilibrium step profiles and, through Monte Carlo simulations, reproduced the clean surface step patterns.

The dynamics associated with atom attachment and detachment should be altered when a step is exposed to an etchant species at elevated temperature. In particular, the overall detachment rate should be enhanced when the surface is etched. Comparison of post-etch step patterns to those for clean surface steps shows much increased kink length and frequency. Significantly, we demonstrated that, under the moderate etching that takes place at $700 \mathrm{~K}$, the step patterns can be described using the same atomic-level interactions deduced for the clean surface, with account taken for the fact that etching biases the step profile by increasing the detachment rate relative to the attachment rate [51].

Rioux et al. [11] analyzed the step roughness as a function of flux at $900 \mathrm{~K}$. They showed that both $S_{\mathrm{A}}$ and $S_{\mathrm{B}}$ steps roughened with increasing flux. Step roughening was taken to reflect the ability of the surface to heal the damage induced by etching at high temperature, i.e., the ability of diffusing $\mathrm{Si}$ adatoms to reattach. Those experiments were done at fixed fluence so that the amount of time that the sample had at elevated temperature was reduced as the flux increased. The complexities and all of the details evident in the patterns at $900 \mathrm{~K}$ involved cannot be easily described because processes associated with terrace etching and chain regrowth are important. In particular, pits formed in front of an advancing step will be incorporated at the step, and the roughness of the step will be influenced by such breakthrough. Likewise, the development of overhangs via kink formation becomes an important mechanism that precludes the use of a solid-on-solid description.

\subsection{Dimer chain}

The islands observed in Fig. 2 are regrowth structures of similar appearance to growth structures observed in Si homoepitaxy [51-54]. In etching, islands form from $\mathrm{Si}$ atoms released when desorption events remove one atom of a dimer and destabilize the now-unpaired atom. Fig. 5(a) shows several dimer chains and several etch pits on a surface etched at $800 \mathrm{~K}$. The chains run perpendicular to the terrace dimer rows. Within a chain the bright elliptical features are individual dimers. Examples of chains with even, E, and odd, O, numbers of dimers are labeled. Extensive counting demonstrates that chains with an odd number of dimers greatly outnumber those 


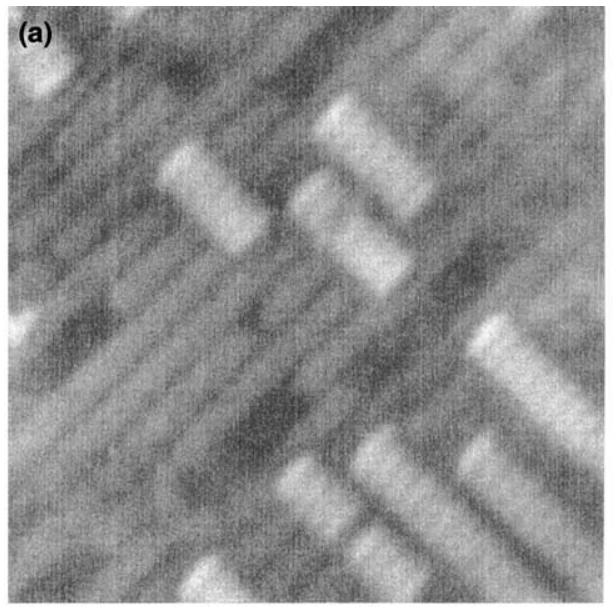

(b) o dimer chain layer - terrace layer - second layer

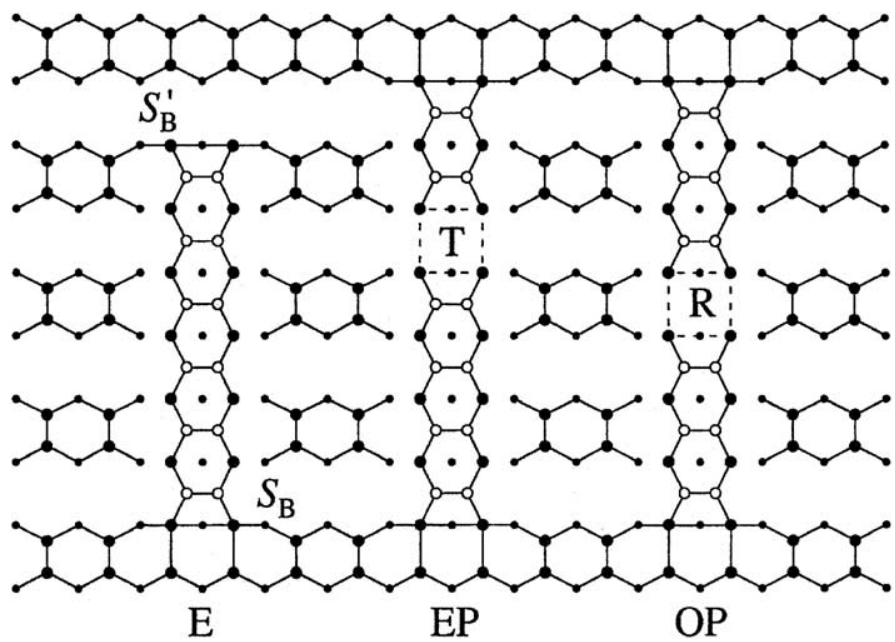

Fig. 5. (a) Occupied states image showing even- and odd-numbered dimer chains, labeled $\mathrm{E}$ and $\mathrm{O}$. Chain termination is favored in the troughs between dimer rows of the terrace, resulting in odd-length chains. Other structures such as even-length paired segments, EP, separated by a single missing dimer are thought to originate as odd-length chains. (b) Schematic showing rebonded $S_{\mathrm{B}}$ and nonbounded $S_{\mathrm{B}}^{\prime}$ steps. Configurations for even- and odd-length paired segments, EP and OP, are shown to result from removal of dimers that were atop troughs, $\mathrm{T}$, or dimer rows, $\mathrm{R}$.

with an even number for etching between 700 and $900 \mathrm{~K}$. Most even-length chains appear in pairs, EP, separated by a single missing dimer. This suggests that they are segments of an odd-length chains that were split.

Removal of a dimer from an odd-length chain could produce either two evenlength segments or two odd-length segments. Analysis of many images like Fig. 5(a) 
revealed nearly equal numbers of EP and OP segments, demonstrating that neither the trough nor the row sites were preferentially vacated. Finally, odd-length chains with end dimers that were both atop rows of the underlying terrace were rare. We find that only $\sim 5 \%$ of the chains are of the isolated even-number type after etching at 700 and $800 \mathrm{~K}$. For $3 \times 1$ steady-state surface produced at $900 \mathrm{~K}$, the fraction of even-length chains increased to $\sim 10 \%$. The increase can be attributed to the coordinated etch mechanism responsible for $3 \times 1$ domain formation. In particular, these dimer chains do not develop via standard growth process of the sort reflected by etching at 700 and $800 \mathrm{~K}$ but through terrace fragmentation. The dominance of oddlength chains is explained by the energetics of step formation of rebonded $S_{\mathrm{B}}$ steps that are energetically favored over nonbonded or $S_{\mathrm{B}}^{\prime}$ steps [40].

Statistical distributions for odd-length chains are shown in Fig. 6 for steady-state etching at 700,800 , and $900 \mathrm{~K}$. The distributions have been normalized to their peak values for comparison. Chains for the $900 \mathrm{~K}$ case were predominantly from $3 \times 1$ domains which cover much of the surface, while those from surfaces etched at 700 and $800 \mathrm{~K}$ were from $2 \times 1$ domains. A shift of the peaks in Fig. 6 to a greater length with increasing temperature is evident, and the tails of the distributions represent increasing fractions of the total count.

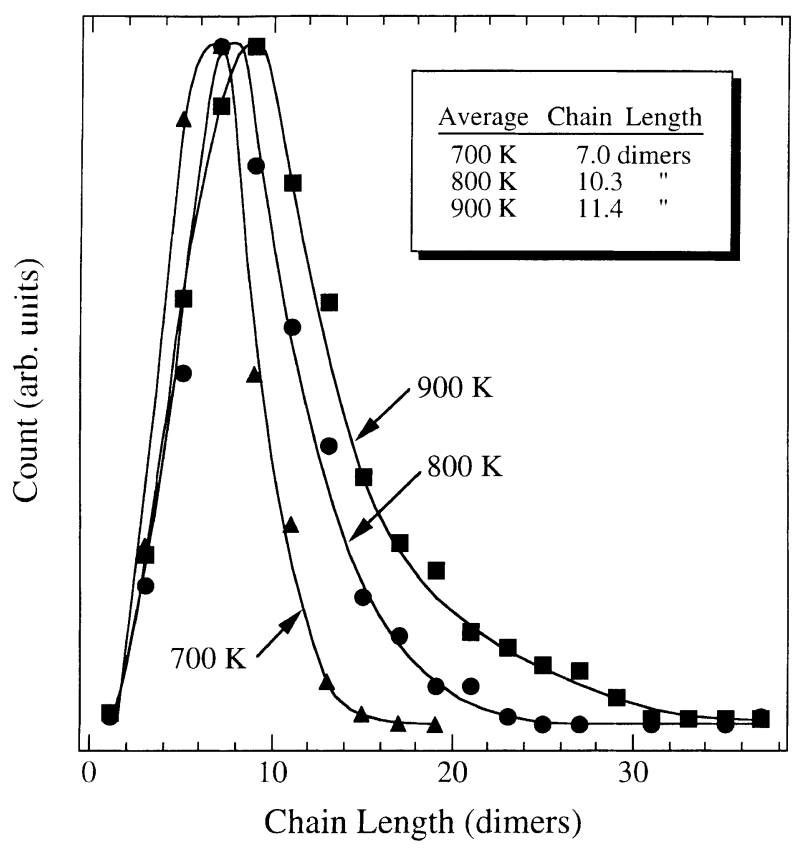

Fig. 6. Dimer chain length distributions for steady-state surfaces etched at 700, 800, and $900 \mathrm{~K}$. Chain lengths are dependent on the amount of $\mathrm{Si}$ available for growth, and this increases with temperature. Chain lengths also depend on the growth mechanism. It is pure regrowth on terraces at $700 \mathrm{~K}$ but it includes regrowth on existing chains at $800 \mathrm{~K}$. At $900 \mathrm{~K}$, the distribution extends to longer lengths. In this case, the chain lengths are limited by the size of the $3 \times 1$ domains. 
Factors contributing to dimer chain lengths include temperature-dependent effects such as the amount of material available for regrowth, the mechanisms involved in their formation, and the average distance between etch pits. The length of chains on surfaces etched at $700 \mathrm{~K}$ is limited by the amount of material available for regrowth. For surfaces etched at $800 \mathrm{~K}$, however, the chain length is limited by the distance between etch pits. For etching up to $800 \mathrm{~K}$, a larger amount of $\mathrm{Si}$ is liberated with increasing temperature, and this counteracts the tendency of coarsening and etching to shorten the chains. At $900 \mathrm{~K}$, chain formation relies on spontaneous etch processes that create $3 \times 1$ domains from $2 \times 1$ terraces. Therefore, the chain length is limited by the domain size.

\section{Atomic processes in etching of $\operatorname{Si}(100)$}

Thermally activated etching involves $\mathrm{SiX}_{2}$ desorption, with $\mathrm{X}=\mathrm{Cl}, \mathrm{Br}$, or I ( $\mathrm{F}$ will be treated in Section 11), and this requires the formation of the adsorbed precursor state $\mathrm{SiX}_{2}(\mathrm{a})$. Desorption is envisioned to start from a configuration in which one halogen adatom is bonded to each $\mathrm{Si}$ atom of a surface dimer. To transfer a halogen atom from one $\mathrm{Si}$ atom to its partner in a dimer requires the breaking of the dimer bond. This prepares a $\mathrm{SiX}_{2}$ unit that can attempt to desorb by overcoming a desorption barrier. Because of the energies involved, most of the time the $\mathrm{SiX}_{2}$ unit will decay by passing back the halogen atom. When an etching event occurs, a $\mathrm{SiX}_{2}$ molecule is released into the gas phase. Associated with it is the transfer of the nowunpaired bystander $\mathrm{Si}$ atom onto the terrace. This creates a DV and provides a $\mathrm{Si}$ atom for regrowth on the terrace. This sequence of events is depicted in Fig. 7.

It is clear that DVs form, that $\mathrm{Si}$ atoms move onto the terrace, and that $\mathrm{SiX}_{2}$ desorbs. However, the exact order of the atomic events was explored by De Wijs et al. [55] who examined them with calculations of the relevant barriers and lifetimes. They suggested that the key event was not the desorption of $\mathrm{SiX}_{2}$ but rather it was the escape of the bystander. This escape would destabilize the $\mathrm{SiX}_{2}$ unit and allow it to make many more attempts than it otherwise could. They concluded that $\mathrm{SiCl}_{2}$ (a) formation was a necessary first step on a surface dimer, $2 \mathrm{SiCl}(\mathrm{a}) \leftrightarrow \mathrm{SiCl}_{2}(\mathrm{a})+\mathrm{Si}(\mathrm{a})$, but that it underwent the reverse reaction too quickly to account for the experimental rate of desorption. They argued that the reverse reaction would be frustrated, however, if a single atom vacancy could be formed adjacent to it by the escape of the $\mathrm{Si}$ (a) atom. The possibility that vacancy formation could play a crucial role in desorption prompted us to undertake a series of STM studies to test the model and to explore the implications.

One way we devised to determine the correct sequence of events was to measure the rate of desorption from surfaces that were dosed with halogens and then heated. If desorption is the key, then the rate should increase steadily with halogen coverage as $\mathrm{SiX}_{2}$ formation on the surface becomes more and more likely. If escape of the bystander is the key, then the rate should go through a maximum at an intermediate coverage because having too much halogen on the surface would prevent transfer of $\mathrm{Si}$ onto the surface. Results indeed suggest that the maximum desorption yield 

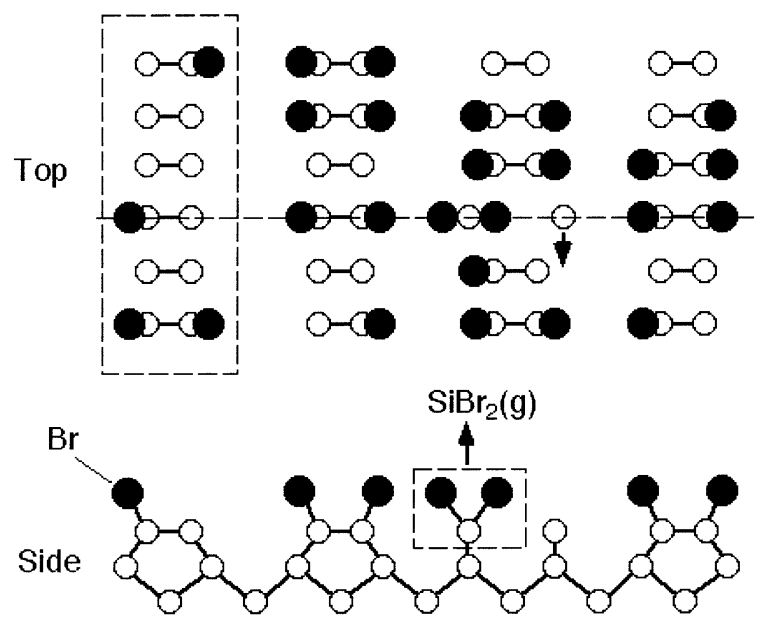

Fig. 7. Schematic indicating $\mathrm{Br}$ (or $\mathrm{Cl}$ or $\mathrm{I}$ ) adatoms on $\mathrm{Si}(100)-2 \times 1$, viewed from the top and the side. The boxed area at the left represents the low concentration regime with $\mathrm{Br}$ bonded both singly and pairwise to Si dimers. Pairwise occupancy dominates if adatom diffusion is made possible by mild annealing. During an etching event, a halogen atom is transferred to form an adsorbed $\mathrm{SiBr}_{2}$ (a) unit, depicted at the right, and thermal desorption produces $\operatorname{SiBr}_{2}(\mathrm{~g})$. Associated with etching is the release onto the surface of the unpaired $\mathrm{Si}$ atom (arrow at right).

occurs when the amount of halogen is about $75 \%$ of a monolayer, as will be discussed in more detail in Section 9 after presentation of the experimental results. An implication is that saturating the surface reduces the etch rate, in contrast to what was generally assumed.

In these experiments, we exposed clean $\mathrm{Si}(100)-2 \times 1$ to $\mathrm{Cl}_{2}$ and $\mathrm{Br}_{2}$ at room temperatures and then subjected the samples to a thermal ramp to activate etching, as in TPD. We showed that the etch rate reached a maximum when the surface was $\sim 75 \%$ saturated, in contrast to what would be expected from the conventional picture of etching where the rate should increase steadily with coverage. Using the results of de Wijs et al., we modeled the desorption rate in terms of the energetics of the various configuration involving surface $\mathrm{Si}$ and $\mathrm{Cl}$. By making the connection with surface concentration, we showed that the frustration of the intermediate step in the overall process, the creation of the vacancy, results in the down-turn in etch rate at high coverage $[56,57]$.

A similar conclusion comes from the experiments recently carried out by Yates and co-workers [58]. During TPD, an extremely fast $\mathrm{SiCl}_{2}(\mathrm{~g})$ desorption is observed on $\mathrm{Cl}$-saturated surfaces. They concluded that an additional high energy kinetic step is necessary to initiate the $\mathrm{SiCl}_{2}$ (g) desorption since there are not any bare Si sites. As soon as this process turns on, the desorption of $\mathrm{SiCl}_{2}(\mathrm{~g})$ occurs catastrophically via the low energy desorption process. The coverage-dependent desorption kinetics were deduced based on the $\mathrm{SiCl}_{2}(\mathrm{~g})$ desorption rate.

Fig. 8 shows the structure for $\mathrm{Cl}-\mathrm{Si}\left(\begin{array}{lll}1 & 0 & 0\end{array}\right)-2 \times 1$ for a sample that had been etched by heating to $800 \mathrm{~K}$ for $10 \mathrm{~min}$, starting with an initial coverage of $0.62 \mathrm{ML}$. The 

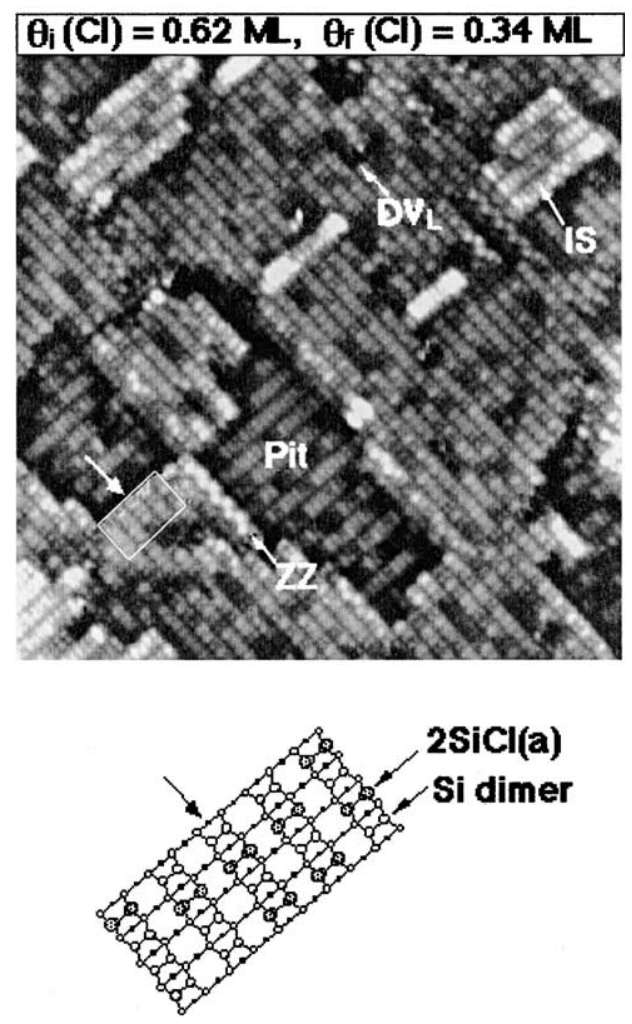

Fig. 8. High resolution image of $\mathrm{Cl}-\mathrm{Si}(100)-2 \times 1$ for a sample that was exposed to $\mathrm{Cl}_{2}$ at room temperature to produce a coverage of $0.62 \mathrm{ML}$ and then heated to $800 \mathrm{~K}$ for $10 \mathrm{~min}$ to activate etching. The initial and final coverages $(0.62$ and $0.34 \mathrm{ML})$ were determined by direct counting of Cl-occupied dimers. Etching and diffusion of vacancies and $\mathrm{Si}$ adatoms produced pits that were one atomic layer in depth, DV lines, $\mathrm{DV}_{\mathrm{L}}$, and regrowth islands, IS. The box beneath the image shows the chemisorption pattern for the corresponding area in the image. Cl-occupied dimers, $2 \mathrm{SiCl}(\mathrm{a})$, appear grouped across dimer rows but not along the rows. $\mathrm{Cl}$ atoms occupy the two $\mathrm{Si}$ dangling bonds of a dimer and produce two bright features with a darker center line. Undifferentiated elliptical structures correspond to bare $\mathrm{Si}$ dimers. $\mathrm{ZZ}$ represents bare $\mathrm{Si}$ dimers at pit boundaries that are buckled. Room temperature imaging; $-2.5 \mathrm{~V} ; 0.2 \mathrm{nA}$.

dimer rows in the main layer run from upper left to lower right while those in the etch pits and the regrowth islands, IS, were rotated by $90^{\circ}$. Dimers with $\mathrm{Cl}$ are darker than those that were $\mathrm{Cl}$-free, in contrast to $\mathrm{Br}-\mathrm{Si}\left(\begin{array}{ll}10 & 0\end{array}\right)$. Fig. 8 provides direct evidence for the dominance of dimers with $\mathrm{Cl}$ atoms bonded to each dangling bond, $2 \mathrm{SiCl}$ (a) [59-62] because these dimers had two bright features with a dark center area. The overall final $\mathrm{Cl}$ concentration was $0.34 \mathrm{ML}$, but the atoms were not distributed uniformly on the three layers. Instead, the coverage of the main layer was $0.39 \mathrm{ML}$, that in the pits was $0.33 \mathrm{ML}$, and that on the regrowth islands was 0.30 ML. There were no singly occupied dimers, but there were dimer rows that are 
$\mathrm{Cl}$-free adjacent to pit or at the edge of regrowth islands. Such dimers can be stabilized in a buckled configuration, $\mathrm{ZZ}$.

The area highlighted by the box of Fig. 8 is depicted beneath the image to identify dimers that were pairwise-occupied. Their arrangement indicates that dimer-dimer interactions favor chains extended across the dimer rows. Occupancy of adjacent dimers along a dimer row was not favored. These patterns are evident for $\mathrm{Cl}$ concentrations below $\sim 0.3-0.4 \mathrm{ML}$, but they were lost at higher coverage. In contrast, low-coverage results for $\mathrm{Br}-\mathrm{Si}\left(\begin{array}{lll}1 & 0 & 0\end{array}\right)$ indicated a $\mathrm{c}(4 \times 2)$ configuration in which occupancy of adjacent pairs was not favored along either direction. The tendency to form $(c 4 \times 2)$ structures is even more pronounced for $\mathrm{I}-\mathrm{Si}\left(\begin{array}{lll}1 & 0 & 0\end{array}\right)$ [63].

\section{Coverage-dependent etch morphologies}

Halogen etching occurs at high temperature against a background where adatoms move, steps fluctuate, and vacancies diffuse. The diffusion barriers that control such movement are sensitive to the halogen concentration and, accordingly, the surface structure reflects that concentration. Etch pits can grow on a terrace via the etching of dimers at their perimeters, but they can also grow by the capture of vacancies and they can coarsen via vacancy exchange. Halogens also dictate the diffusivity of Si adatoms, and this controls the formation of Si regrowth islands. Previous presented results of surface morphologies correspond to etching when the concentration was high and constant so that vacancy diffusion was small. Here, we emphasize profiles achieved when the concentration is decreased and when vacancy and step edge diffusion becomes relevant.

The images of Figs. 9(a)-(c) and 8 were all obtained after heating to $800 \mathrm{~K}$ for 10 min. They present different morphologies due to the concentrations of $\mathrm{Cl}$ during etching. Fig. 9(a) shows the morphology produced by etching when the surface $\mathrm{Cl}$ concentration was always high, $0.8 \mathrm{ML} \leqslant \theta(\mathrm{Cl}) \leqslant 1.0 \mathrm{ML}$. Under these conditions, pits developed on the terraces and their growth favored elongation along the dimer row direction to produce $\mathrm{DV}$ lines, $\mathrm{DV}_{\mathrm{L}}$, that were a single dimer wide. Pit growth across dimer rows occurred when there was a branching event, and several pits can be seen that were two or three dimers in width. The energy differences that dictated such growth were discussed in Section 6. Etching also roughened the steps and caused them to retreat $[37,64]$, and bright chains of Si grew on the Cl-rich terraces. These regrowth structures were up to 13 dimers in length, and most were a single dimer row in width.

Fig. 9(b) was obtained for a surface etched with an intermediate $\mathrm{Cl}$ coverage, 0.42 $\leqslant \theta(\mathrm{Cl}) \leqslant 0.77 \mathrm{ML}$. It shows large, single-layer-deep etch pits and regrowth features up to 7 rows in width. Occasionally, there were also dimer chains, labeled $\mathrm{RG}^{\prime}$, on regrowth islands. The changes in pit sizes and shapes reflect the fact that DVs had greater mobility and could coalesce or be captured at pits. The $S_{\mathrm{B}}$ step roughness was enhanced by the diffusion of vacancies to the step. Etching from $\theta(\mathrm{Cl})=0.77 \mathrm{ML}$ gave a surface with the greatest area density of pits. As we show below, the etch rate is fastest in this coverage regime. 

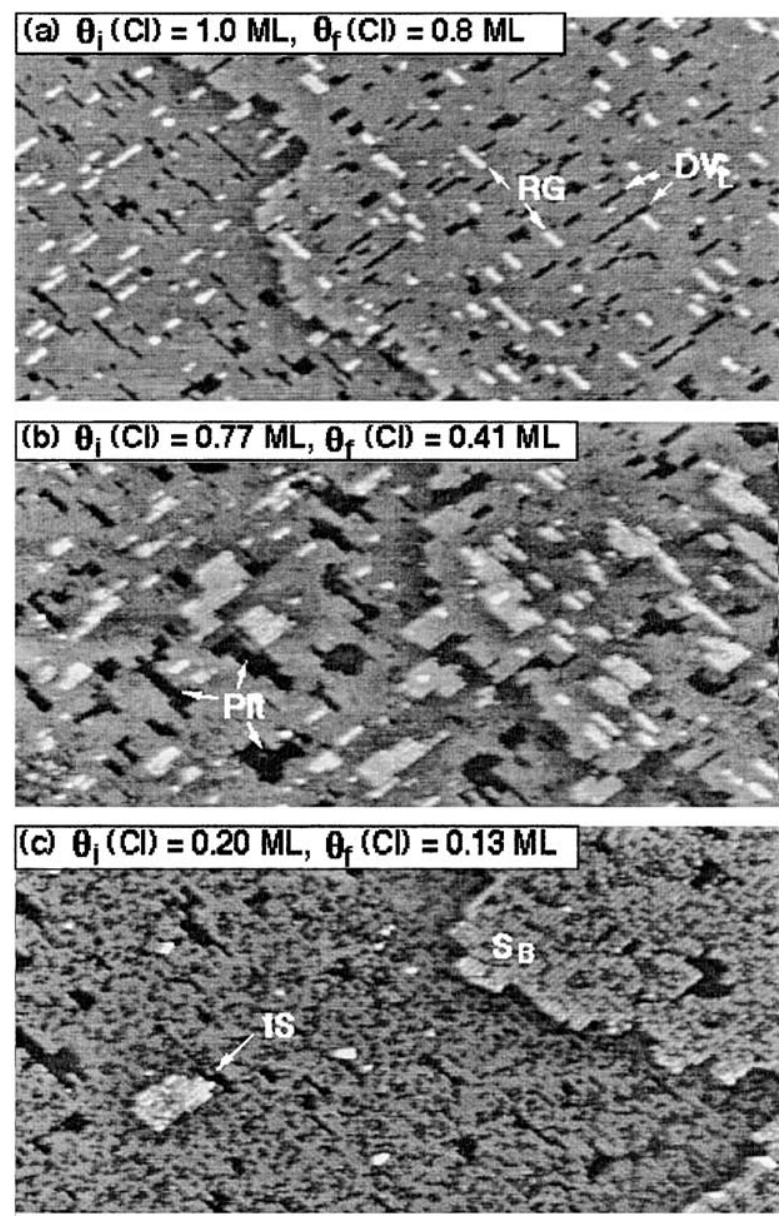

Fig. 9. Occupied state images for dosed surfaces that were heated to $800 \mathrm{~K}$ for $10 \mathrm{~min}$. Room temperature imaging; $-2.5 \mathrm{~V} ; 0.2 \mathrm{nA}$. (a) Etching at high coverage produced DV lines, $\mathrm{DV}_{\mathrm{L}}$, regrowth chains of one or two dimers in width, RG, and step retreat. Vacancy and $\mathrm{Si}$ adatom diffusion was hindered by the high $\mathrm{Cl}$ concentration. (b) Etching at intermediate coverage produced DVs that grew by further etching and by vacancy capture. With reduced $\mathrm{Cl}$, the $\mathrm{Si}$ adatoms were more mobile and large regrowth islands developed. The areal pit density was the greatest in this coverage range. (c) Etching at low coverage had about the same etch rate as in (a) but the final morphology was different because of the reduced $\mathrm{Cl}$ concentration on the terraces. In this image, the intermediate-gray features reflect dimers with $\mathrm{Cl}$ adatoms.

Fig. 8 is representative of atomic resolution images of the etching morphology obtained with an even lower concentration, $0.34 \leqslant \theta(\mathrm{Cl}) \leqslant 0.62 \mathrm{ML}$. The pit in the center of the image crosses eight dimer rows and is derived from 230 missing dimers. Such extended pits dominate the surface. DV lines such as the one labeled $\mathrm{DV}_{\mathrm{L}}$ were relatively few, and single-dimer vacancies were even less frequent.

Fig. 9(c) shows the result of etching at low coverage, $0.13 \leqslant \theta(\mathrm{Cl}) \leqslant 0.20 \mathrm{ML}$, where the etch rate was considerably reduced. There were no long single-width 
regrowth chains and, instead, there were large islands. Note that the intermediategray areas represent residual $\mathrm{Cl}$ that was visible in this image because of good tip conditions.

\section{Vacancy creation and etching pathway}

As noted in Section 7, two distinctly different sequences of events have been proposed to describe $\operatorname{Si}\left(\begin{array}{lll}1 & 0 & 0\end{array}\right)-2 \times 1$ etching. For both, the initial configuration involves two halogens bonded to two atoms of a dimer, 2SiX(a). For both, there is an isomerization reaction that produces an adsorbed $\mathrm{SiX}_{2}(\mathrm{a})$ species and a silicon atom $\mathrm{Si}(\mathrm{a})$ that has two dangling bonds and is adjacent to the $\mathrm{SiX}_{2}$ (a) species.

In the traditional sequence of events, thermal activation leads to the desorption of $\mathrm{SiCl}_{2}$ (a) and $\mathrm{Si}(\mathrm{a})$, which is taken to be a bystander, is left to fend for itself. Since it is no longer stabilized by dimerization, $\mathrm{Si}(\mathrm{a})$ escapes as $\mathrm{Si}(\mathrm{s})$ and readily diffuses away [12]. Hence, $\mathrm{SiCl}_{2}(\mathrm{a})+\mathrm{Si}(\mathrm{a}) \rightarrow \mathrm{SiCl}_{2}(\mathrm{~g})+\mathrm{Si}(\mathrm{a})-E_{2} \rightarrow \mathrm{DV}+\mathrm{Si}(\mathrm{s})$ where $E_{2}$ is the activation energy of $\mathrm{SiCl}_{2}$ desorption.

As mentioned in Section 7, de Wijs et al. [55,65] recently challenged this picture. Based on first principles local density functional calculations, they determined that the formation of $\mathrm{SiCl}_{2}$ (a) in Eq. (1) costs $E_{1} \sim 1.4 \mathrm{eV}$ and that the desorption barrier for $\mathrm{SiCl}_{2}$ (a) was very high, $E_{2} \sim 3.2 \mathrm{eV}$. Moreover, they estimated that $\mathrm{SiCl}_{2}(\mathrm{a})$ would decay back to the monochloride configuration within $\sim 50 \mathrm{~ns}$ at $850 \mathrm{~K}$ because the reverse barrier was only $0.7 \mathrm{eV}$. Hence, few molecules would be in the $\mathrm{SiCl}_{2}(\mathrm{a})$ state long enough to desorb, and surface etching would be small compared to what is observed.

To account for etching, de Wijs et al. proposed that an etching event was started by the escape of the bystander at a cost of only $E_{3}=0.03 \mathrm{eV}$ and an activation energy of $0.6-1 \mathrm{eV}$. The escaped silicon atom, $\mathrm{Si}(\mathrm{s})$, is mobile, and the likelihood of returning to the vacancy is negligible. Hence, $\mathrm{SiCl}_{2}(\mathrm{a})$ is deprived of its decay pathway and it had a much greater chance of thermal desorption, even though the desorption barrier was still high, $\sim 3.1 \mathrm{eV}$. The key step in this model is the thermally activated creation of a vacancy adjacent to $\mathrm{SiCl}_{2}(\mathrm{a})$.

To test the validity of the model, we determined the amount of material removed from the terraces for surfaces like those of Figs. 8 and 9 as a function of the initial surface concentration. The traditional model would predict that the etch rate would increase with initial coverage since the number density of $2 \mathrm{SiX}$ (a) units would increase. However, if the escape of the bystander were the crucial step, then the rate should decrease because suitable terrace sites would be occupied by $\mathrm{Cl}$ atoms at high coverage.

Fig. 10 summarizes the results of quantitative analysis of surfaces etched with $\mathrm{Cl}$ and $\mathrm{Br}$. The data points demonstrate that overall rates for both species increase with coverage until $\sim 0.75 \mathrm{ML}$ but then decrease. For $\mathrm{Cl}$, the decrease is dramatic, and the etch rate for a saturated surface is small. For Br, the decrease is less profound be-

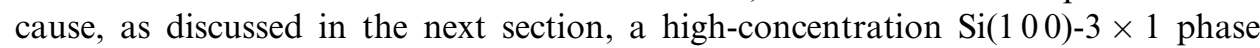
introduces a pathway not considered here. 


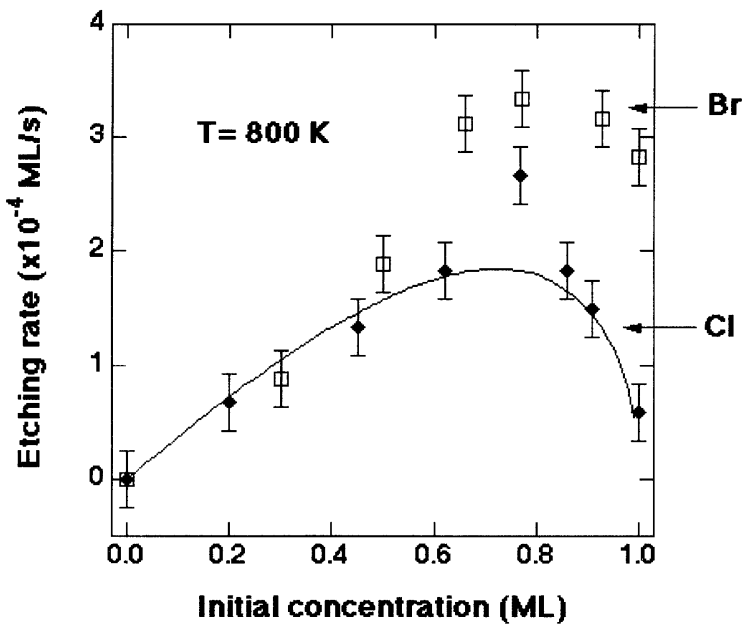

Fig. 10. Plot of the etch rate for $\mathrm{Si}\left(\begin{array}{ll}0 & 0\end{array}\right)$ as a function of the initial concentration of $\mathrm{Cl}$ and $\mathrm{Br}$, where the rate is the amount of $\mathrm{Si}$ removed divided by the time. All samples were dosed at room temperature and heated to $800 \mathrm{~K}$ for $10 \mathrm{~min}$. Analysis was done on wide terraces far from steps. The maximum etch rate occurs at $\sim 0.77 \mathrm{ML}$. The solid line was obtained from Eq. (3) and was based on the relative concentrations of the configurations defined in Fig. 11, with no adjustable parameters.

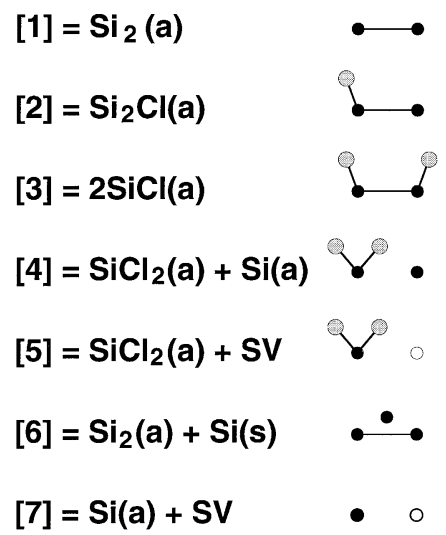

Fig. 11. Seven surface configurations for $\mathrm{Cl}$ adatoms on $\mathrm{Si}(100)-2 \times 1$. The areal densities of these species are interrelated via their relative binding energies. [f] is established by vacancy creation adjacent to a $\mathrm{SiCl}_{2}$ (a) unit, and the etch rate is proportional to its concentration. The formation of [f] is inhibited by site blocking at high halogen coverage.

To relate the etching rate, $\mathrm{d} c / \mathrm{d} t$, to the concentration of $\mathrm{Cl}$ on the surface, $\theta(\mathrm{Cl})$, we must consider the possible surface configurations, the relative energies that dictate the partitioning of $\mathrm{Cl}$, and the desorption barriers for the precursor states. The relevant configurations are depicted in Fig. 11. Etching occurs from the conventional 
intermediate state [d], though at a negligible rate, and from [f], the new intermediate state.

There are two conservation equations or mass balances. For $\mathrm{Si}$ atoms at the surface, we have $[\mathrm{a}]+[\mathrm{b}]+[\mathrm{c}]+[\mathrm{d}]+[\mathrm{f}] / 2+3[\mathrm{~h}] / 2+[\mathrm{j}] / 2=1$. The $\mathrm{Cl}$ coverage is given by $\theta(\mathrm{Cl})=[\mathrm{b}] / 2+[\mathrm{c}]+[\mathrm{d}]+[\mathrm{f}]$. The dominant species that can be observed with STM are surface dimers with and without $\mathrm{Cl}$, namely [a] and [c], which is consistent with the energetically favored occupancy of species [c] over [b], [55]. Then, we adopt the following simplifications $[\mathrm{a}]+[\mathrm{c}]=1$ for $\mathrm{Si}$, and $\theta(\mathrm{Cl})=[\mathrm{c}]$. We can assume that the number of $[\mathrm{h}]$ configurations is equal to $[\mathrm{f}]+[\mathrm{j}]$, because Si adatoms must have been created on an ideal surface by dimer splitting.

The energies associated with producing these configurations, and thereby their populations, were developed by de Wijs et al. $[55,65]$. If we assume that the surface is in dynamic equilibrium, then concentrations can be related through their Arhenius dependence on temperature $T$. Thus,

$$
\begin{aligned}
& {[\mathrm{d}] /[\mathrm{c}]=\exp \left(-E_{1} / k_{\mathrm{B}} T\right),} \\
& {[\mathrm{f}][\mathrm{h}] /[\mathrm{a}][\mathrm{d}]=\exp \left(-E_{3} / k_{\mathrm{B}} T\right),} \\
& {[\mathrm{c}][\mathrm{j}] /[\mathrm{a}][\mathrm{f}]=\exp \left(E_{4} / k_{\mathrm{B}} T\right)}
\end{aligned}
$$

Finally, the etching rate, $\mathrm{d} c / \mathrm{d} t$, is proportional to the concentration of intermediate states, [f], which depends on the halogen concentration $[\mathrm{c}]=\theta(\mathrm{Cl})$. Solving for [f] in terms of the energies and concentrations, we obtain $\mathrm{d} c / \mathrm{d} t=\mathrm{k}[\mathrm{f}]$, so that

$$
\begin{aligned}
\mathrm{d} c / \mathrm{d} t= & k\left\{\exp \left[-\left(E_{1}+E_{2}\right) / k_{\mathrm{B}} T\right)\right] \theta(\mathrm{Cl})[1-\theta(\mathrm{Cl})] / \\
& {\left.\left[1+\exp \left(E_{4} / k_{\mathrm{B}} T\right)[1-\theta(\mathrm{Cl})] / \theta(\mathrm{Cl})\right]\right\}^{1 / 2}, }
\end{aligned}
$$

where $k$ is a constant.

Eq. (3) introduces a connection between the surface concentration and the ability to create a vacancy that would facilitate etching. According to it, the reduction in the reaction rate is simply described as site blocking. Using it, we obtained the solid line in Fig. 10, with $k=18$, where the etch rate peaks at $\theta(\mathrm{Cl})=0.72 \mathrm{ML}$. The agreement with experiment for $\mathrm{Cl}$ is compelling, and we conclude that vacancy creation is a key step in etching reactions. Note that the only relevant parameter that would change the exact form of $\mathrm{d} c / \mathrm{d} t$ is $E_{4}$, the energy gained in transferring two $\mathrm{Cl}$ atoms from $\mathrm{SiCl}_{2}$ far from the vacancy site, but there would still be reaction inhibition and a turndown, independent of $E_{4}$.

There are important implications that follow from these observations. First, etching at low temperature will be suppressed because the surface concentration is high and vacancy reaction is unlikely. Second, increasing the flux in steady-state etching can increase $\theta$ and reduce, rather than increase, the etch rate. Third, an increase in $\theta$ can inhibit the dominant pathway and increase the relative importance

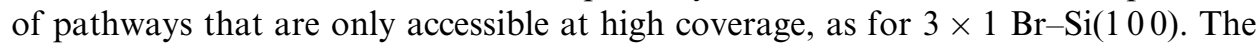
existence of multiple pathways accessed as $\theta$ changes in a desorption study complicates the analyses in terms of activation energies. 


\section{Consequences of $3 \times 1$ reconstruction}

The agreement between experiment and the site-blocking model of Eq. (3) is good for $\mathrm{Cl}$ but not so good for $\mathrm{Br}$. This suggests that a new reaction pathway has been accessed at high coverage for $\mathrm{Br}$, one not included in Eq. (3). Indeed, the already mentioned phase transformation from $2 \times 1$ to $3 \times 1$ for $\mathrm{Br}-\mathrm{Si}\left(\begin{array}{lll}1 & 0 & 0\end{array}\right)$ gives rise to removal of atomic rows rather than dimer rows. It also introduces a new stabilization step that increases the lifetime of $\operatorname{SiBr}_{2}(\mathrm{a})$ and enhances its desorption.

Studies with atomic resolution STM have made it possible to conclude that there

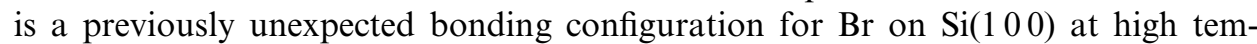
perature and coverage. The normal pathway described above occurs under conditions where at most one $\mathrm{Br}$ atom bonds to each $\mathrm{Si}$ dangling bond. This concentration can be increased by raising the temperature to $900 \mathrm{~K}$. At this temperature, there is phase transformation to a $3 \times 1$ structure where rows of $\mathrm{SiBr}_{2}$ units alternate with dimer rows of $\mathrm{SiBr}$ ( $\mathrm{Si}$ with a single $\mathrm{Br}$ atom). When Chander et al. [9] investigated etch morphologies as a function of $\mathrm{Br}$ dose at $800-900 \mathrm{~K}$, they observed pits and DV lines for low and intermediate doses. However, when the surface concentration reached a critical value, they discovered that single-atom-wide vacancy rows were also produced. They concluded that the $2 \times 1$ surface reconstructed to $3 \times 1$ at $800-900 \mathrm{~K}$ where rows of dimers with $\mathrm{SiBr}$ bonding alternated with $\mathrm{Si}$ monomers with $\mathrm{SiBr}_{2}$ bonding and desorption eliminated the $\mathrm{SiBr}_{2}$ rows. More recently Weaver and co-workers [57] determined that with the appropriate conditions of temperature- and coverage-limited $3 \times 1$ formation could also be found for chlorine.

To investigate the transformation further, samples with different initial $\mathrm{Br}$ coverage were heated to $800 \mathrm{~K}$ for $10 \mathrm{~min}$ and then the morphology was examined. Fig. 12(a) shows dimer vacancy lines, limited amounts of regrowth, and a roughened $S_{\mathrm{B}}$-type step for $0.11 \leqslant \theta(\mathrm{Br}) \leqslant 0.3 \mathrm{ML}$. Here, dimers with $\mathrm{Br}$ adatoms appeared brighter than those that were bare. Small areas showed $\mathrm{c}(4 \times 2)$ ordering of $\mathrm{Br}$ adatoms, in contrast to the alignment of Cl-bonded dimers of Fig. 8. Etching at intermediate coverage, $0.43 \leqslant \theta(\mathrm{Br}) \leqslant 0.77 \mathrm{ML}$, produced larger pits and regrowth islands, Fig. 12(b). Regrowth structures are labeled $\mathrm{RG}_{1}$ if their axis is parallel to the dimer row and they reside between dimer rows. They are labeled $R_{2}$ if they grow as epitaxial layers. There were also a few atom vacancy lines. Finally, etching at $0.78 \leqslant \theta(\mathrm{Br}) \leqslant 1 \mathrm{ML}$ produced a very different morphology, Fig. 12(c) with both atom vacancy lines and DV lines. The area enclosed in the box depicted in Fig. 12(d), where two atomic vacancy lines are shown and where residual $\mathrm{Br}$ is present on the dimer rows. From these coverage-dependent studies, we conclude that atomic vacancy lines were produced when $\theta(\mathrm{Br})$ exceeded $\sim 0.7 \mathrm{ML}$ and that their density increased to a maximum at 1.0 ML. Moreover, they always appeared in pairs separated by dimer rows. Analysis of these images made it possible to determine the etch rate, as discussed above for $\mathrm{Cl}$. From Fig. 10, the etch rate for $\mathrm{Si}-\mathrm{Cl}$ at $800 \mathrm{~K}$ is close to that for $\mathrm{Si}-\mathrm{Br}$ for low and intermediate coverage, but etching from the $3 \times 1$ reconstruction accounted for the much higher etch rate at high coverage for $\mathrm{Si}-\mathrm{Br}$ compared to $\mathrm{Si}-\mathrm{Cl}$. 

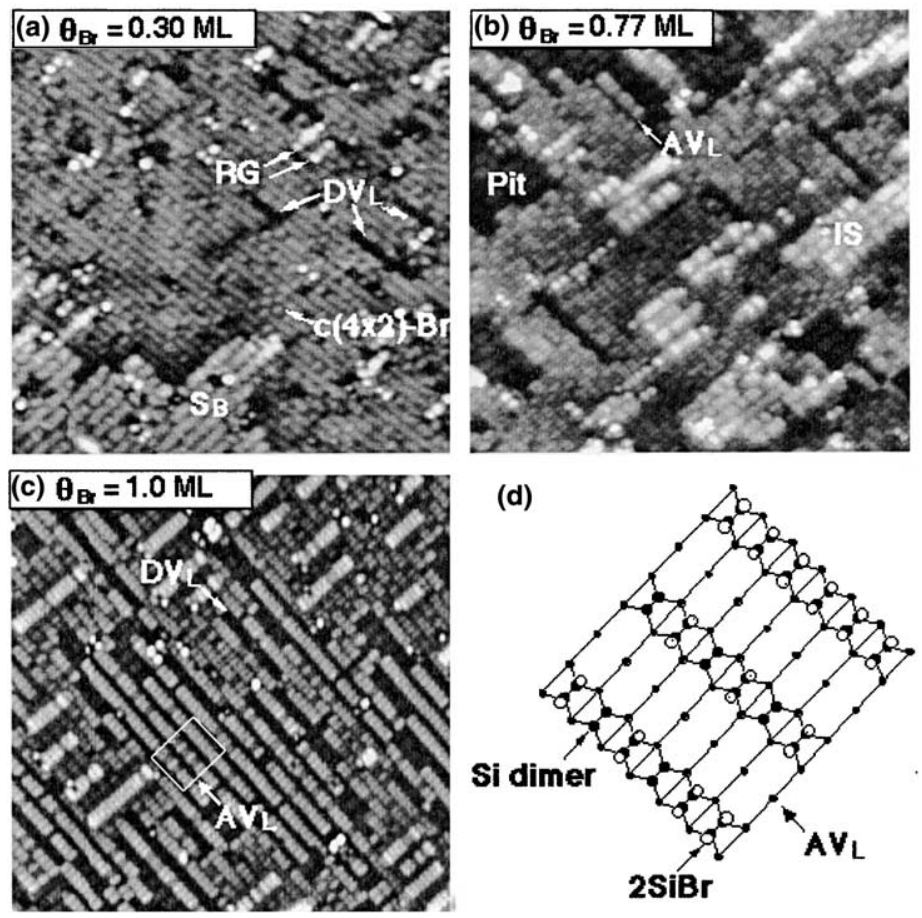

Fig. 12. Images showing the dependence of morphology on surface concentration for $\mathrm{Br}-\mathrm{dosed} \operatorname{Si}\left(\begin{array}{lll}1 & 0 & 0\end{array}\right)$ $2 \times 1$ that was heated to $800 \mathrm{~K}$ for $10 \mathrm{~min}$. Room temperature imaging, sample bias $-2.5 \mathrm{~V} ; 0.2 \mathrm{nA}$. (a) Etching at low coverage produced DV lines, $\mathrm{DV}_{\mathrm{L}}$, and regrowth features, RG. Terraces were decorated with $\mathrm{c}(4 \times 2)-\mathrm{Br}$ chemisorption features. (b) Etching an intermediate coverage resulted in a maximum material removal and surface irregularity as pits formed and grew, DVs coalesced, and regrowth islands developed against a decreasing background of mobile $\mathrm{Br}$. (c) Etching at high coverage produced a unique morphology related to the $3 \times 1$ reconstruction. Atom vacancy lines, $\mathrm{AV}_{\mathrm{L}}$, were produced by $\mathrm{SiBr}_{2} \mathrm{de}-$ sorption, as depicted in Fig. 13. These patterns were robust because single atom vacancies were not mobile. (d) A schematic of the boxed region of (c) showing two atomic vacancy lines, dimer rows, and residual $\mathrm{Br}$.

The high-coverage desorption pathway can be visualized from Figs. 13(a)-(d). Starting from a locally saturated area, Fig. 13(a), thermal activation drives the isomerization reaction to produce one $\mathrm{Si}(\mathrm{a})$ and one $\mathrm{SiX}_{2}(\mathrm{a})$. The forward and reverse rates are indicated as $k_{i+}$ and $k_{i-}$. If we assume that the local halogen density does not fluctuate, then there are four configurations that can be established on neighboring dimer rows. The one of interest is depicted in Fig. 13(b), namely $[\mathrm{X}-\mathrm{Si}-\mathrm{X}+\mathrm{Si}, \mathrm{Si}+\mathrm{X}-\mathrm{Si}-\mathrm{X}]$ because the two $\mathrm{Si}$ atoms can dimerize, as shown in Fig. 13(c). Dimerization lowers the energy of the intermediate state and establishes a substantial barrier for the reverse isomerization reaction. Dimerization then serves the same function as the escape of the bystander discussed above because it increases the $\mathrm{SiX}_{2}$ (a) lifetime. At $800 \mathrm{~K}$, the structure depicted in Fig. 9(c) acts as the nucleus for the $3 \times 1$ phase and the transition state for desorption, Fig. 9(d). This nucleus will grow as chains of $\operatorname{SiX}_{2}(\mathrm{a})$ units and bare Si dimers elongate along [0 11 1]. This 
(a)

2SiX(a), where $X=C l, B r$

(a)

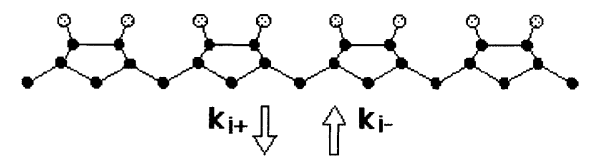

(b)

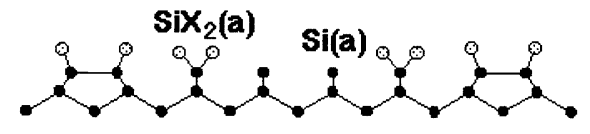

(c)

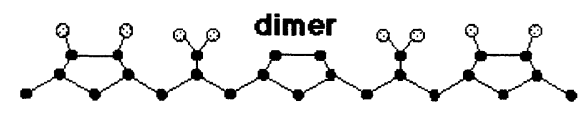

\section{$\operatorname{six}_{2}(g)$}

(d)

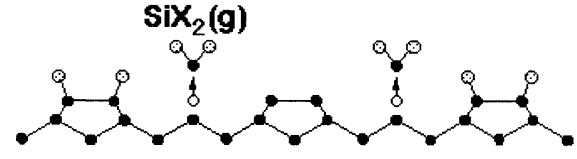

Fig. 13. Systematic showing the proposed formation and desorption pathway for $\mathrm{Br}-\mathrm{or} \mathrm{Cl}-\mathrm{Si}(100)$ at high coverage. (a) The initial configuration at saturation coverage with all dangling bonds occupied by $\mathrm{X}=\mathrm{Cl}$ or $\mathrm{Br}$. (b) Isomerization reaction that produces $\mathrm{SiX}_{2}$ (a) and a bystander, $\mathrm{Si}(\mathrm{a})$. If this occurs on adjacent dimer rows, then the $\mathrm{Si}(\mathrm{a})$ atoms can dimerize to produce (c). This restricts the reverse reaction to (a) and the rate $k_{i-}$ is small. The lifetime of $\mathrm{SiX}_{2}$ (a) is increased and desorption is more likely. Atom vacancy lines are produced in pairs, separated by a single dimer row, as observed.

model has the advantage that it does not require mass transport of halogens from other portions of the surface or halogen uptake from a continuous source.

\section{Three-dimensional pitting in etching $\operatorname{Si}(100)-2 \times 1$ with $\mathrm{F}$}

As reviewed in the previous sections, STM has provided the connection between desorption products and structural changes for thermally activated processes involving $\mathrm{Cl}$ and $\mathrm{Br}$. Equivalent connections have not been established for $\mathrm{F}$ etching, though theoretical studies have simulated the reaction of fluorine on Si surfaces [6669] and low temperature etching is envisioned as involving the formation of a multilayer of fluorosilyl species [2]. However, structural consequences of thermally activated reactions of fluorine for initial $\mathrm{F}$ coverages below $\sim 0.5 \mathrm{ML}$ have been recently addressed [70].

As for other halogens, $\mathrm{F}$ atoms react with the silicon dangling bonds to form $\mathrm{F}-\mathrm{Si}(100)-2 \times 1[71,72]$. Etching is then linked to the desorption of $\mathrm{SiF}_{2}$ that establishes a dimer vacancy. What is different here is that $\mathrm{F}$ atoms can also react with the dangling bonds of exposed second-layer $\mathrm{Si}$ atoms to form volatile $\mathrm{SiF}_{2}$, and then multilayer defects at low surface concentration are produced. Such double-layer pitting allows us to describe corrosive surface reactions and the roughening that is known to occur when $\mathrm{Si}$ is exposed to $\mathrm{F}$. 

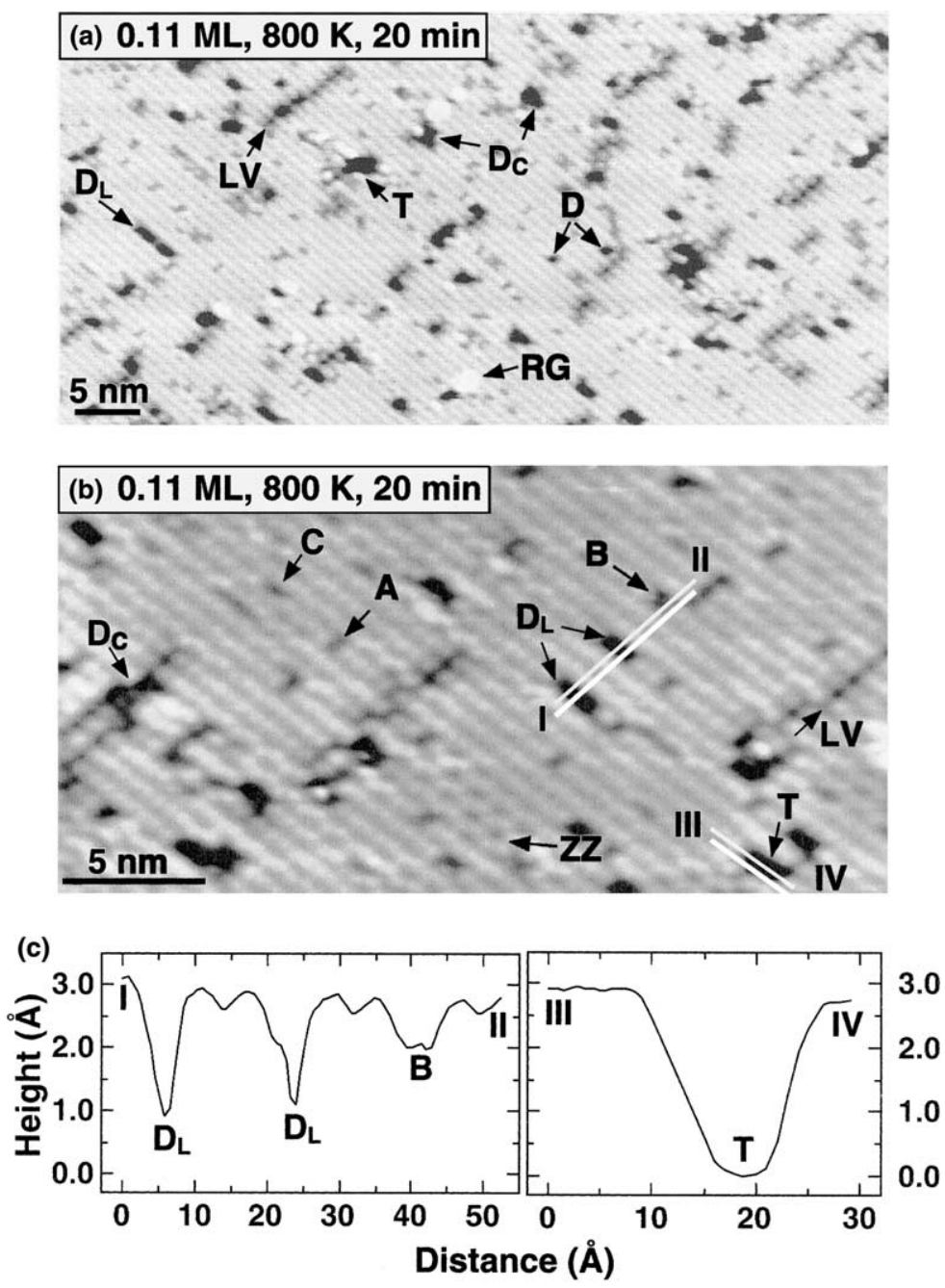

Fig. 14. (a) and (b) STM images showing F-induced changes to $\operatorname{Si}\left(\begin{array}{lll}1 & 0 & 0\end{array}\right)-2 \times 1$. The samples were dosed at room temperature and heated to induce $\mathrm{SiF}_{2}$ desorption. Top layer defects include single DVs, A, pairs of DVs, B, and line vacancies that assembled from mobile vacancies, LV. Double layer defects appeared singly, $\mathrm{D}$, or as lines or complexes, $\mathrm{D}_{\mathrm{L}}$ and $\mathrm{D}_{\mathrm{C}}$. $\mathrm{RG}$ identifies Si regrowth. $\mathrm{T}$ calls attention to triple layer defects. Other symbols are defined in the text. Sample bias $-2 \mathrm{~V}$, current $0.2 \mathrm{nA}$. (c) Line scan I-II crosses a top layer B-type defect and two double layer $\mathrm{D}_{\mathrm{L}}$ defects. Scan III-IV crosses a triple layer defect elongated along the dimer row direction.

Figs. 14(a) and (b) show the morphology achieved after a surface dosed with 0.11 $\mathrm{ML}$ of $\mathrm{F}$ was heated at $800 \mathrm{~K}$ for $20 \mathrm{~min}$ and then quenched. Many different types of defects can be distinguished. The most common defect is a top-layer DV, A-type. They tend to occur in lines that run perpendicular to the dimer row, and they are gray in the images. Such line vacancies, LVs, are pinned by multilayer pits because of 
the robust geometric structure produced by contact. Defects tend to stabilize the buckling motion of nearby dimers, producing asymmetric dimers that are visible as bright protrusions centered on alternating atoms along the rows, labeled ZZ. Fig. 14 also reveals bright structures on the main terrace that represent regrowth features, $\mathrm{RG}$, built up from Si released as part of vacancy creation events, as for etching with other halogens. C-type defects appear in occupied state images as two bright protrusions on one side of a dimer row [73].

What is unique for $\mathrm{F}$ is that Si removal is no longer limited to the top layer and, somehow, second layer removal becomes a common occurrence. The darkest areas correspond to multilayer pits. They are labeled D if they are a double unit deep. Those labeled $D_{L}$ are extended along the dimer row. Fig. 14(b) identifies $D_{L}$-type vacancies in which up to 4 dimers have been removed from the top layer. The profile along lines I-II in Fig. 14(c) shows that the $\mathrm{D}_{\mathrm{L}}$ 's are two layers deep. It also shows that B-type defects are derived from two top layer DVs. Defects labeled $\mathrm{D}_{\mathrm{C}}$ correspond to double layer complexes that span more than one row. Also identified are Ttype defects and profiles, such as III-IV, demonstrate that they are three-layers deep.

The question to ask is: what is special about fluorine that causes such a profound change in the etching pathway? Much of the answer can be found by considering top layer vacancies and the bonding sites available to monovalent halogens. From the STM results, it is clear that the key structure is the B-type double-DV. These structures are relatively rare during the earliest stages of etching, in part because vacancies tend to be captured in LVs before they coalesce into B-type defects. Once formed, however, they become a channel to the second layer.

The top and side views of Figs. 15(a) and (b) show a B-type vacancy with $\mathrm{F}$ atoms bonded to dangling bonds of second layer Si atoms. If there were no $\mathrm{F}$, the exposed Si atoms would likely form long $\sigma$-bonds with second layer neighbors, I with III or II with IV. This rebonded structure is stable when exposed to $\mathrm{Cl}$ or $\mathrm{Br}$ at all temperatures investigated. When exposed to $\mathrm{F}$, however, our experimental results show that the B-type defect convert to double layer pits. Nakayama and Weaver [70] proposed that the new reaction pathway involves $\mathrm{SiF}_{2}$ (a) formation, as in Fig. 15(b), followed by desorption that leads to second-layer etching, Fig. 15(c). Third-layer pitting is also possible as the pit grows.

Steric and chemical considerations make it possible to rationalize why secondlayer etching is possible for $\mathrm{F}$, but not for $\mathrm{Cl}$ or $\mathrm{Br}$. First-principles calculations $[65,74]$ indicate that the Si-halogen bond length on the top layer is much shorter for $\mathrm{SiF}_{2}$ (a) than for $\mathrm{SiCl}_{2}(\mathrm{a}), 1.62 \AA$ vs. $2.13 \AA$, in agreement with bond lengths of 1.55 and $2.02 \AA$ for $\mathrm{SiF}_{4}$ [75]. The side views of the double dimer pit shows the implications for adsorbed $\mathrm{F}$ and $\mathrm{Cl}$. Since the bulk $\mathrm{Si}-\mathrm{Si}$ bond length is $2.35 \AA$, $\mathrm{Cl}$ atoms bonded at I-III or II-IV would be able to form bridge bonds with two Si atoms. Chlorine bridge bonding is not favored in defect-free $\operatorname{Si}(100)-2 \times 1$ [59] because it requires the breaking of dimer bonds, but a configuration like that of Fig. 15(b) is robust.

Competing with vacancy-assisted etching then is a new channel associated with the structure in Fig. 3(a), and there are two possible pathways. In both, two F atoms must reach the exposed $\mathrm{Si}$ atoms of the second layer, bonding to sites I-IV of 


\section{(a) double dimer vacancy}

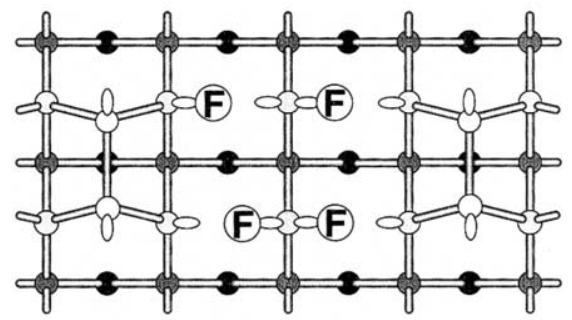

(b) side view
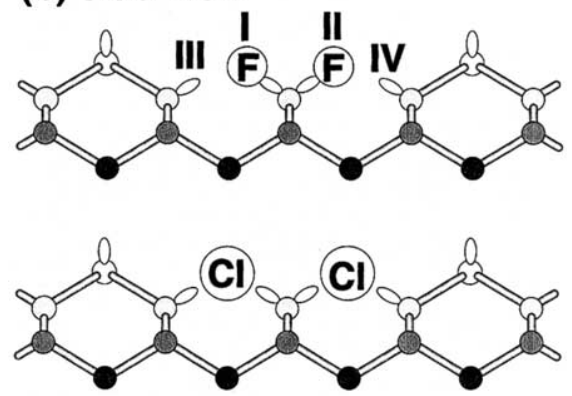

(c) double layer pitting

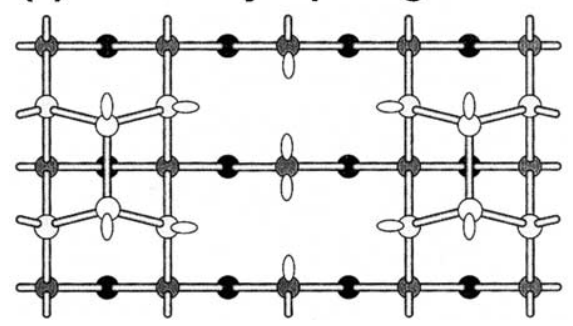

Fig. 15. (a) and (b) Schematics of $\mathrm{Si}\left(\begin{array}{lll}1 & 0 & 0\end{array}\right)-2 \times 1$ with a B-type double $\mathrm{DV}$ with $\mathrm{F}$ and $\mathrm{Cl}$ adatoms. $\mathrm{For} \mathrm{Cl}$, the $\mathrm{SiCl}_{2}$ (a) structure is not favored, and second-layer etching does not occur. For F, second-layer etching results in a double layer pit, as shown in (c).

Fig. 15(b). In this configuration, one can imagine resonant $\mathrm{F}$ hopping between sites I and III or sites II and IV. Significantly, activation of $\mathrm{SiF}_{2}$ (a) can occur without the $\sim 1.4 \mathrm{eV}$ barrier associated with $\sigma$-bond breaking in top layer isomerization reactions [55]. Moreover, the effective lifetime of the $\mathrm{SiF}_{2}$ (a) state is enhanced by the retention of $\mathrm{F}$ within the pit, and there is not need to create a vacancy of the sort needed on the top layer.

A second pathway is suggested by the high local concentration of $\mathrm{F}$ associated with the double dimer pit, and this would involve back-bond insertion. Insertion could be envisioned from Fig. 15(b) if a F transfers from site IV into the backbond without involving site II. Though it costs a $\sigma$-bond (as for the isomerization reac- 
tion), the new state would be weakly bound to the surface and desorption would follow. It has been argued that bond insertion accounts for the corrosive etching of $\mathrm{Si}$ and the formation of a fluorosilyl. Either pathway could account for the high yield of second-layer etching while insertion could make the link to the fluorosilyl layer formed after exposure in the temperature regime when $\mathrm{SiF}_{2}(\mathrm{a})$ desorption is low.

\section{2. $\mathrm{Si}(1111)$ etching}

Despite the complexity involved in $\mathrm{Si}\left(\begin{array}{lll}1 & 1 & 1\end{array}\right)$ etching, the spatially ordered arrangement of surface sites makes its study possible. The $7 \times 7$ reconstruction offers many different desorption energies for the different sites of $\mathrm{Si}$ atoms in the outermost adatom layer. The open, loosely bound adatom layer is readily attacked by halogens. The Si adatoms can be completely stripped from the surface while the rest of the surface structure remains intact. The exposed $\mathrm{Si}\left(\begin{array}{lll}1 & 1 & 1\end{array}\right)$ rest-layer changes its structure in response to the presence of halogen atoms. Terraces convert into a bulk-terminated surface that are stabilized by halogen atoms. This structural transformation is accomplished with extra $\mathrm{Si}$ atoms that are most easily supplied by steps and, for this reason, bulk domains tend to appear near step edges.

Heating to $500-650 \mathrm{~K}$ during $\mathrm{Br}_{2}$ exposure leads to conversion to the $1 \times 1$ structure after $\geqslant 3 \mathrm{~mA}$ s fluence and suitable annealing (15-20 min) with minimal etching, as confirmed by TPD results on Si surfaces [76]. During this process, bilayer islands are formed that have a characteristic dimension of 10-30 $\mathrm{A}$. They reflect the accumulation of atoms that had originally constituted the adatom layer. Adatoms not contributing to island formation are consumed by healing dimer rows of the reconstruction. After exposures in this temperature range, we observed no evidence for etching such as terrace pit formation or step edge roughening. Equivalent results have been discussed by Boland and Villarrubia [22,23] and by Owman and Martensson [80] for $\mathrm{Cl}-$ and $\mathrm{H}-\mathrm{Si}\left(\begin{array}{lll}1 & 1\end{array}\right)-7 \times 7$, respectively. As noted, the driving force for the $7 \times 7$ reconstruction is lost when the $\mathrm{Si}$ dangling bonds are saturated by halogens or hydrogen.

\subsection{Si(111) etching at $900 \mathrm{~K}$}

Fig. 16 shows the effects of 1.2 and $6 \mathrm{~mA}$ s fluence on terrace etched at $900 \mathrm{~K}$. The bright spots represent residual $\mathrm{Si}$ adatoms, labeled $\mathrm{A}$. The exposed rest atom layer is atomically resolved as a local $1 \times 1$ structure, saturated by $\mathrm{Br}$. The outlined unit cell shows the persistence of the $7 \times 7$ periodicity. The dark lines in the image correspond to cell boundaries defined by dimer rows of the reconstruction. Atomic resolution images of the Br-bonded are similar to those for Cl-Si(llll) [23], H-Si(1 111 ) [77], and $\mathrm{NaOH}$ wet-etched $\mathrm{Si}\left(\begin{array}{lll}1 & 1 & 1\end{array}\right)$ [20]. Also evident are sites where $\mathrm{Si}$ atoms are missing from the restatom layer, labeled V. These vacancies are almost exclusively located near unit cell edges where there are dimer rows in the next layer. This indicates that $\mathrm{Br}$ weakens $\mathrm{Si}$ backbonds and that removal near dimer rows is easiest where $\mathrm{Si}-\mathrm{Si}$ bonds are strained. 

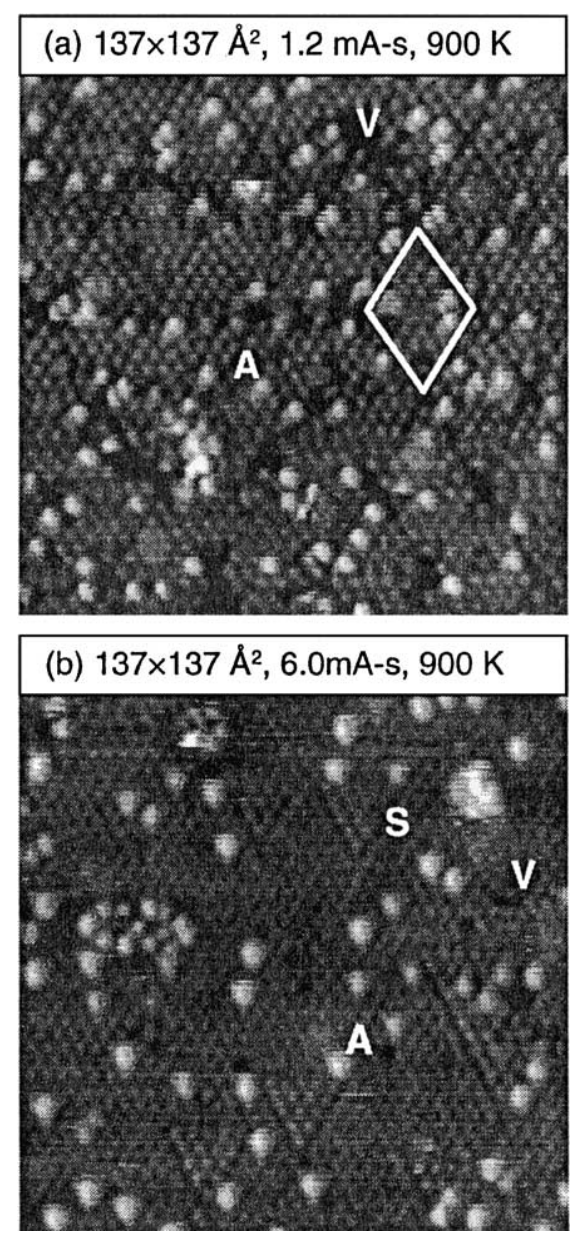

Fig. 16. Bromine etching of $\operatorname{Si}(111)-7 \times 7$ at $900 \mathrm{~K}$ as a function of $\mathrm{Br}_{2}$ fluence for terrace areas far from steps (flux $20 \mu \mathrm{A}$ ). (a) After $1.2 \mathrm{~mA} \mathrm{~s}$, most of the adatoms, A, were gone, leaving the restatom layer with Br-bonding that stabilized the local $1 \times 1$-like structure. The dark lines demonstrate the presence of the dimer rows of the original $7 \times 7$ cell. Vacancies in the restatom layer are denoted V. (b) After $6 \mathrm{~mA} \mathrm{~s}$, the terrace has further transformed to a Br-terminated $1 \times 1$ structure although some dimer rows persist and give produce stacking faults $(\mathrm{S})$. The $\mathrm{Br}$ concentration is close to saturation on the terraces; only the steps act as sites where $\mathrm{Br}$ is lost.

Most of the surface of Fig. 16(b) has converted to $1 \times 1$, and only a few stacking faults $(\mathrm{S})$ remain, as observed for a $\mathrm{Cl}$-covered $\mathrm{Si}(111)-7 \times 7$ surface that had been annealed. The atomic scale morphology represented in Fig. 16(b) is unchanged for longer exposures. Hence, continuous etching at $900 \mathrm{~K}$ is characterized by terraces that have Br-terminated $1 \times 1$ character with a few vacancies and residual adatoms. The dominant pathway for Si removal is via step retreat. Despite the abundance of $\mathrm{Br}$, the barrier against pit formation is too great to allow pits to form. 
For $\operatorname{Si}(100)-2 \times 1$, etching is accompanied by the formation of regrowth islands that nucleate from liberated, mobile $\mathrm{Si}$ atoms. For $\mathrm{Si}(111)$, ring-shaped clusters develop during etching at $900 \mathrm{~K}$. They are concentrated near steps where $1 \times 1$ regions are exposed. As shown in Fig. 17(a), the ring structures, denoted R, appear as

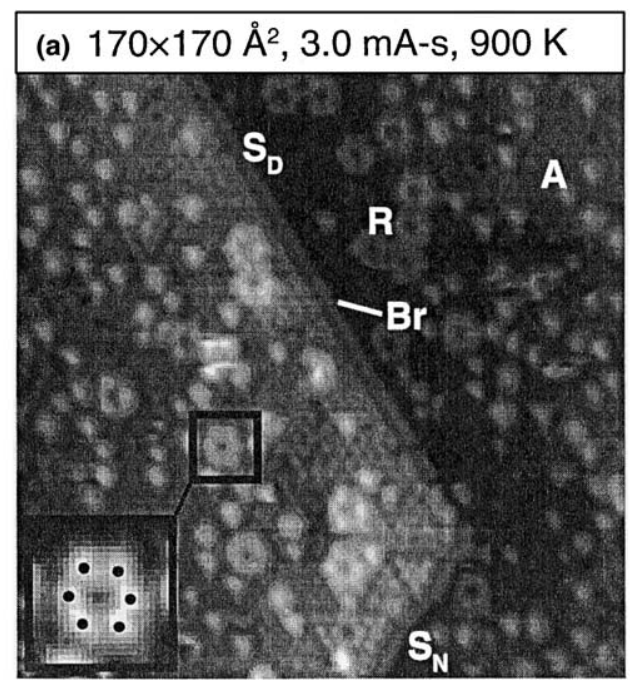

(b)
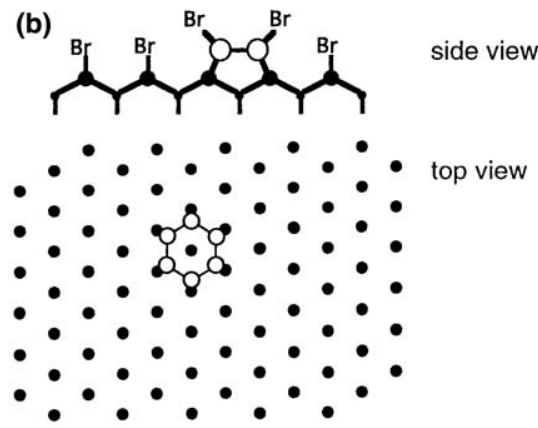

$\mathrm{OSi}$ of ring structure

- Br terminated $\mathrm{Si}(111)$ top layer

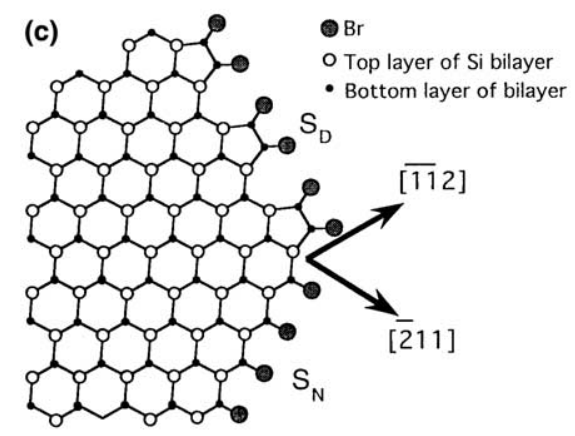

Fig. 17. (a) Bromine etching of $\operatorname{Si}\left(\begin{array}{lll}1 & 1 & 1\end{array}\right)-7 \times 7$ near steps at $900 \mathrm{~K}$ produced regrowth structures, $\mathrm{R}$, that consisted of $\mathrm{Si}_{6}$ rings to which $\mathrm{Br}$ was bonded. The inset shows the ring structure. Rings were more abundant near $S_{D}$ that $S_{N}$ steps. Br termination at step sites indicates the rebonding characteristics of the steps. (b) Schematic of rings as six Si atoms back-bonded to the surface and bonded to neighbors. Fourfold coordination is achieved by Br-termination. (c) Schematic of the two types of close-packed steps on Si(1 11 )- $1 \times 1 . S_{D}$ steps descend along [ $\left.\overline{1} \overline{1} 2\right],[\overline{1} 2 \overline{1}]$, or [2 $\left.\overline{1} \overline{1}\right]$ while $S_{N}$ steps descend toward [ $\left.111 \overline{2}\right],\left[\begin{array}{ll}1 & \overline{2} \\ 1\end{array}\right]$, or

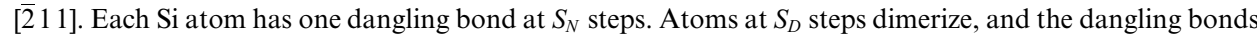
are Br-saturated. Steps of clean $\operatorname{Si}\left(\begin{array}{lll}1 & 1 & 1\end{array}\right)-7 \times 7$ are defined by linear structures that are locally analogous to $S_{D}$ steps due to dimer rows. Kink formation at an $S_{N}$ step involves removal of an atom that had three back bonds. This kink can flow along the step as atoms with only two back bonds are removed. Etching of an $S_{D}$ step is more facile and involves breaking the dimer bond to form $\mathrm{SiBr}_{2}$ with two back bonds. 
atomically resolved six-membered rings on the upper and lower $(1 \times 1)$ terraces. Regrowth structures should reflect the tendency to minimize the number of dangling bonds. Of the possible geometric arrangements, six-membered rings represent the smallest structures that allow four-fold coordination of each Si atom when terminated with Br. Fig. 17(b) depicts the proposed ring structure. Image analysis shows that each ring atom is situated above an $\mathrm{Si}$ atom on a $1 \times 1$ area [78].

Analysis shows that regrowth rings form $\sim 5$ times more frequently near steps

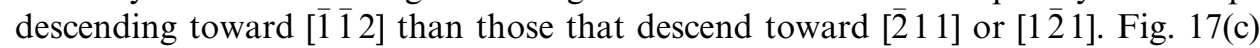
demonstrates that bonding at these two steps is quite different. One type has two dangling bonds and dimerizes along the edge, denoted $S_{D}$, while the other has one dangling bond extending from the step, denoted $S_{N}$. Since the terraces near the two step types are equivalent, the greater density of rings near $S_{D}$ steps indicates greater release of $\mathrm{Si}$ from those steps. Anisotropic etching with easier removal at $S_{D}$ steps relative to $S_{N}$ steps can be rationalized in terms of bond strength arguments [79-81]. Volatile products can be formed more readily at $S_{D}$ edges since there are only two backbonds and $\mathrm{SiBr}_{2}$-like bonding is achieved when the $\sigma$-bond is broken. Volatile species formation at $S_{N}$ steps has a higher activation energy since it requires attack of the three backbonds. Hence, kink formation limits the etching of $S_{N}$ steps. Once an atom is removed from an $S_{N}$ step, however, that kink is readily etched because atoms on both sides of the kink have two dangling bonds. The step profiles revealed that more than $90 \%$ of the step length is comprised of $S_{N}$ steps. This again indicates $S_{N}$ steps are more stable than $S_{D}$ steps.

\subsection{Si(111) etching at $1000 \mathrm{~K}$}

Etching at $1000 \mathrm{~K}$ continuous to involve adatom removal, terrace conversion to a $1 \times 1$ structure, and step retreat, as at $900 \mathrm{~K}$. It differs from etching at $900 \mathrm{~K}$, however, in that bilayer pits with distinctive shapes are also formed and these pits grow laterally. The pit sizes and densities depend on the flux because the flux determines the mean $\mathrm{Br}$ concentration achieved under conditions where there is $\mathrm{Br}$ loss via etching.

Fig. 18 shows images of a surface exposed at $1000 \mathrm{~K}$ to a flux of $20 \mu \mathrm{A}$ for a fluence of $1.6 \mathrm{~mA} \mathrm{~s}$. The surface is characterized by macroscopically irregular steps and bilayer terrace pits, P. The step irregularities are triangular, as at $900 \mathrm{~K}$, but the mean separation is smaller and the deviation from linearity is larger. Pit boundaries are defined locally by three equivalent $S_{N}$ steps, as indicated in Fig. 18(b) and shown schematically in Fig. 18(c). The average pit has a characteristic dimension of $1250 \AA$ (distance from pit apex to base). Remnants of upper-terraces are often observed, having been created during terrace retreat and the possible breakthrough of a pit with a retreating step.

Pits can form when two neighboring vacancies are created in the top layer as, for example, atoms numbered 1 and 2 in Fig. 18(c). This produces an atom with two dangling bonds on the second layer (atom 3 in Fig. 18(c)) so that a volatile $\mathrm{SiBr}_{2}$ unit can form. Subsequently, another top layer atom is vulnerable (atom 4 in Fig. 19(c)). This series of events would produce a bilayer pit with four vacancies 

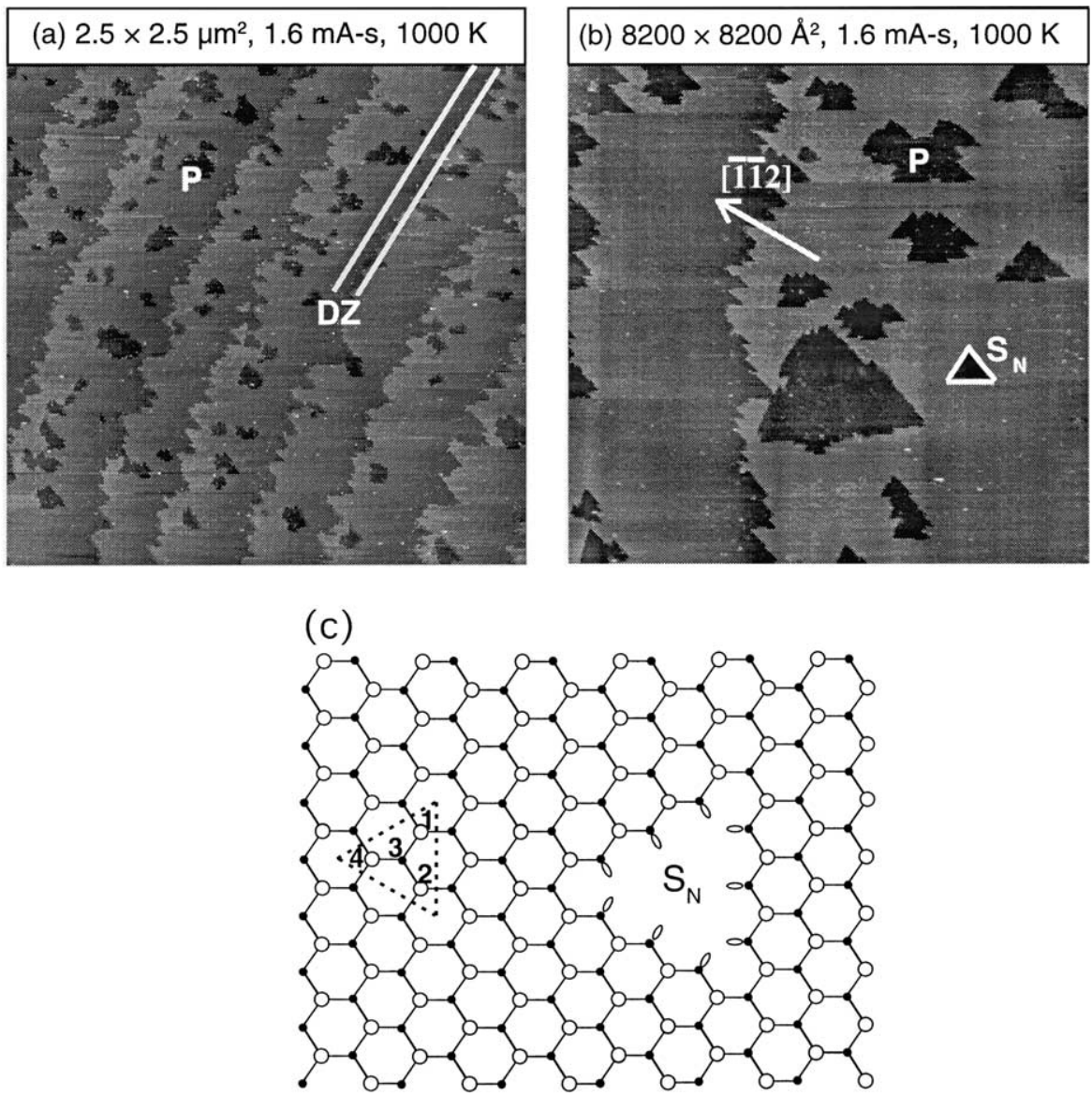

Fig. 18. Bromine etching of $\operatorname{Si}(111)-1 \times 1$ at $1000 \mathrm{~K}$ produces peninsulas at steps and bilayer pits, $\mathrm{P}$, on terraces. (a) Denuded zones, DZ, are regions of reduced pit density near steps. Their width indicates how far the steps have retreated in the time necessary to form a terrace pit. (b) Pits are triangular and are bounded by $S_{N}$ steps. Etching occurs along the step by kink formation and kink flow. (c) Schematic showing a triangular bilayer pit bounded by $S_{N}$ steps. The lengths of the three step edges in the schematic are less than $10 \AA$ and $\operatorname{Si}\left(\begin{array}{llll}1 & 1 & 1\end{array}\right)$ whereas those in the images are much larger. Numbers $1-4$ suggest a sequence of removal events that would lead to the initiation of a bilayer pit bounded by the dashed lines.

bounded by six second-layer atoms each having one dangling bond. Further growth requires removal of a boundary atom from the top layer or the second layer. Doing so would create a kink that exposes two dangling bonds on neighboring $\mathrm{Si}$ atoms. Removal of these kink site atoms is then relatively facile and the kink flows until the pit again assumes a triangular shape bordered by second-layer atoms, each with one dangling bond. The formation of a new kink allows the pit to grow again. At this stage, the pit resembles the one depicted in Fig. 18(c) with boundaries defined by $S_{N}$ edges. 

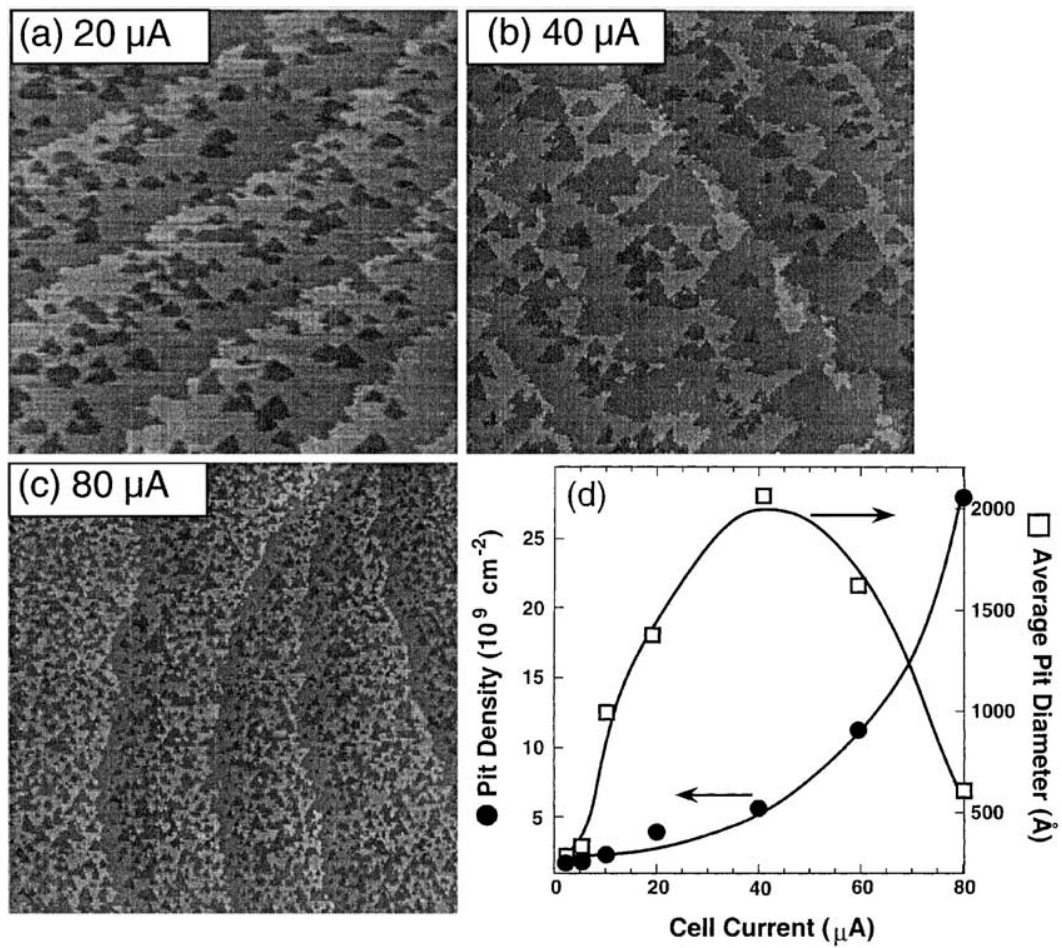

Fig. 19. (a)-(c) Large STM images of Si(1 1 1) etched at $1000 \mathrm{~K}$ to steady-state morphology using the fluxes indicated (images $2 \times 2 \mu \mathrm{m}^{2}$ ). The etchant arrival rate affects local $\mathrm{Br}$ concentrations and the details of etching. (d) Shows the pit density and size as a function of flux. Steady-state Br concentrations increase with flux, and pit formation becomes more probable. Pit sizes also increase with flux due to $\mathrm{Br}$ concentrations and higher-yield step etching. The decline in pit size for fluxes greater than $40 \mu \mathrm{A}$ indicates effects of terrace width.

Fig. 19 shows surfaces etched at $1000 \mathrm{~K}$ with different fluxes to achieve steadystate morphologies (total fluence exceeded $6 \mathrm{~mA} \mathrm{~s}$, but longer exposures gave the same morphology). Inspection shows that the pit shape, size distribution, and density are flux-dependent. For example, a flux of $20 \mu \mathrm{A}$ produced pits with sizes that averaged $1300 \AA$ (density $3.9 \times 10^{9} \mathrm{~cm}^{-2}$ ) while etching with $40 \mu \mathrm{A}$ resulted in an average pit size of $2100 \AA$ (density $5.6 \times 10^{9} \mathrm{~cm}^{-2}$ ). At this point, there was a significant pit-pit and pit-step coalescence, and pitting had started in the next layer. Etching at $80 \mu \mathrm{A}$ yielded much smaller average pit sizes $(600 \AA)$ and a dramatic increase in density $\left(2.8 \times 10^{10} \mathrm{~cm}^{-2}\right)$. A great many pits contacted neighboring pits or steps and the terraces appeared nearly half-eroded. These size and density data are summarized in Fig. 19(d) for fluxes of 2-80 $\mu \mathrm{A}$. It should be noted that pit size and density data can be skewed by pit coalescence. However, the triangular shape of pits enabled us to identify portions of large pits that had originally nucleated as individual pits and then grew together. When such identification was possible, each portion was counted as a separate pit and their sizes were estimated accordingly. 
These results demonstrate that the concentrations needed for attack of Si backbonds on terraces are difficult to achieve at low flux. From Fig. 19(d), the trend is that higher pit densities are the result of increased flux at $1000 \mathrm{~K}$. This is analogous to the situation during growth where, at high flux, the area that the mobile species can sample is small due to the arrival of new species. Supersaturation then increases the likelihood of forming a stable island. This is what is observed for fluxes above $40 \mu \mathrm{A}$. For lower fluxes, $\mathrm{Br}$ is lost via the low energy etching pathways (kink formation and kink flow), and the terrace concentration is not sufficient for pit formation. At very low flux, below $5 \mu \mathrm{A}$, there is also competition with reconstruction of the Si surface, and etching involves different pathways. During local reconstruction, step reordering takes place since there is no edge preference (all edges are $S_{D^{-}}$ like steps on reconstructed areas), and the resulting edge profiles are highly irregular.

The growth of a bilayer pit is governed by the relative rates of kink formation and kink flow. The kink formation rate is proportional to the number of available sites, i.e., pit-edge length, $L$, in addition to the $\mathrm{Br}$ flux $I_{\mathrm{B}}$. When a pit is small, less time is required for a kink to advance across the entire edge than to form a new kink. Experimentally, this is deduced from the average kink-kink separation of $\sim 150 \AA$. At some critical pit edge length, $L_{\mathrm{c}}$, the times required for kink flow and kink formation become equal. If kink formation depends linearly on $I_{\mathrm{B}}$, then pit growth, $\mathrm{d} L /$ $\mathrm{dt}$, is governed by $\mathrm{d} L / \mathrm{d} t \propto I_{\mathrm{B}} L$ for $L \ll L_{\mathrm{c}}$ and $\mathrm{d} L / \mathrm{d} t \propto I_{\mathrm{B}} L_{\mathrm{c}}$ for $L>L_{\mathrm{c}}$. Then, small pits will grow exponentially with time for a given flux. Thus $L \approx \exp \left(I_{\mathrm{B}} t\right)$ since the growth rate increases with $L$. When the pit-edge length exceeds $L_{\mathrm{c}}$, the growth rate loses its dependence on $L$, and growth will become linear with flux and time. In this regime, $L \approx L_{\mathrm{c}}+k L_{\mathrm{c}} I_{\mathrm{B}} t$. This is equivalent to step retreat. Such a dependence on the difference in energy needed to produce a kink and to propagate a kink explains the decrease of average pit size for high Br fluxes shown in Fig. 19(d). Moreover, at high flux as the rate of step retreat increases, the terraces are exposed for a shorter time before they are swept away. In this regime, pit growth is suppressed since step retreat annihilates pits before they achieve sizes corresponding to the exposure time and etch rates. The result is a decrease in average pit size because of reduced growth time.

\subsection{Si(11 1) terrace disordering at $1100 \mathrm{~K}$}

For $\operatorname{Si}\left(\begin{array}{lll}1 & 1 & 1\end{array}\right)$, there is a first order-transition from the $7 \times 7$ to the $1 \times 1$ structure at $\sim 1125 \mathrm{~K}$ [82-84]. Hence, etching at $1100 \mathrm{~K}$ will occur under conditions where there is a tendency to reconstruct to $7 \times 7$ but there is also facile Si removal. For these surfaces, little $\mathrm{Br}$ was observed after cooling, and the morphology is consistent with fast local removal of adatoms.

Fig. 20(a) shows that the steps remain relatively straight and that bilayer pits are not formed after exposure at $1100 \mathrm{~K}$ to $1.2 \mathrm{~mA} \mathrm{~s}$ at $20 \mu \mathrm{A}$. Indeed, the periodicity and roughness of the steps is nearly that of the clean surface. Thus, while steps are susceptible to etching at $1100 \mathrm{~K}$, the high temperature and absence of $\mathrm{Br}$ promotes sufficient Si diffusion that etch-induced step irregularities are eliminated and the step length is minimized. The absence of terrace pits indicates that local $\mathrm{Br}$ concentrations 

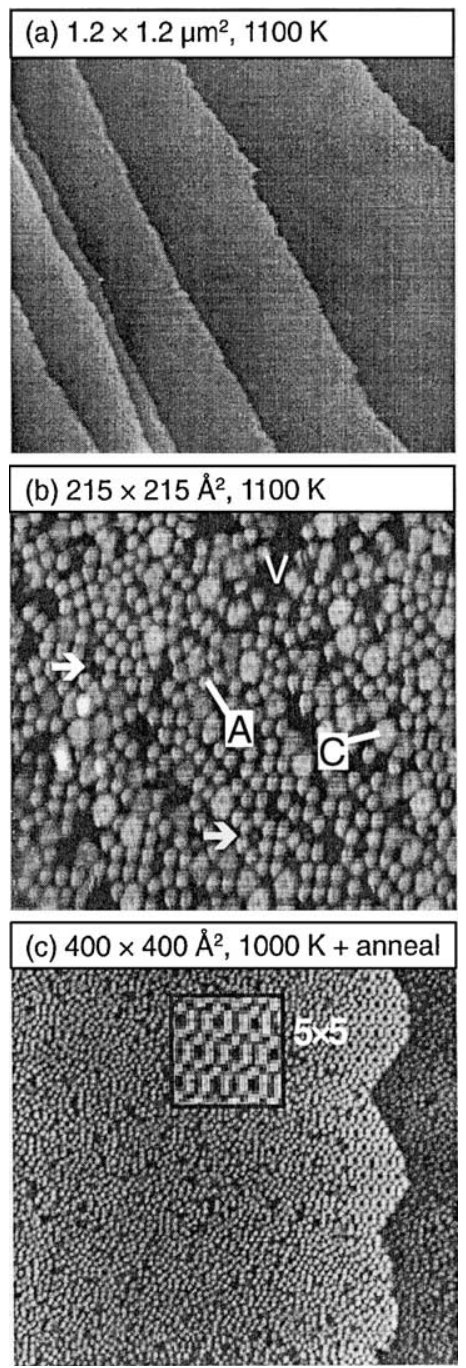

Fig. 20. (a) Large area of an $\mathrm{Si}(111)$ surface etched with $\mathrm{Br}$ at $1100 \mathrm{~K}$ showing that steps remain straight and terrace pitting does not occur. (b) Atomic detail after etching at $1100 \mathrm{~K}$ showing that adatoms, A, are distributed in a disordered fashion although the arrows point to small ordered domains. Vacancy clusters, V, and Si clusters, C, can be seen. (c) Etching to steady-state at $1000 \mathrm{~K}$, as in Fig. 19(a), followed by annealing at $1000 \mathrm{~K}$ for five minutes (without additional $\mathrm{Br}$ ) yields a morphology similar to etching at $1100 \mathrm{~K}$. Ordered domains have nucleated at the steps on upper terraces. A $5 \times 5$ region is indicated and shown in detail in the inset.

needed for pit nucleation cannot be achieved. Results from studies at different flux and fluence at $1100 \mathrm{~K}$ confirm that terrace pits were not formed.

The expanded scale image of Fig. 20(b) shows adatoms (A), vacancies (V), and small clusters $(\mathrm{C})$. The adatoms seem randomly distributed, but small $7 \times 7$ and 
$5 \times 5$ domains can be seen occasionally. The surface then reflects etching with the tendency to reconstruct to reduce the dangling bond density. The persistence of intact corner holes indicated by arrows in Fig. 20(b) suggests that the corner holes act as seeds for the growth of domains. Significantly, the largest domains (up to 150 $\AA$ ) were concentrated at step edges, consistent with the fact that reconstructed areas nucleate at steps for clean surfaces.

Fig. 20(c) shows a surface that was initially etched to steady-state morphology with $20 \mu \mathrm{A}$ at $1000 \mathrm{~K}$, as in Fig. 2(a), and then annealed for $5 \mathrm{~min}$ at $1000 \mathrm{~K}$ after the $\mathrm{Br}$ source was turned off. The resulting morphology can be compared to that of Figs. 20(a) and (b). Interestingly, structures equivalent to those produced by etching at $1100 \mathrm{~K}$ are present after annealing at $1000 \mathrm{~K}$ a surface etched at $1000 \mathrm{~K}$. Steps are macroscopically straight but showed segments of [1 $\overline{2} 1],[\overline{2} 11]$, and [ $\overline{1} 1 \overline{1} 2]$ edges, and no bilayer pits were observed. Terrace adatoms showed no long range ordering, but $5 \times 5$ and $7 \times 7$ domains had nucleated on upper terraces at step edges. A $5 \times 5$ domain is evident in Fig. 20(c) and is detailed in the inset. No $\mathrm{Br}$ was detected on the adatom layer. Substantial diffusion was accomplished and healing of pits and reordering of steps was promoted as residual $\mathrm{Br}$ was lost during the annealing.

\section{Conclusions}

The morphologies for etched $\operatorname{Si}(100)-2 \times 1$ with bromine and chlorine reflect temperature-dependent etch product evolution, diffusion, and surface reactivity. Studies have emphasized morphologies associated with $\operatorname{Si}(100)-2 \times 1$ with large terraces and single height steps. The dominant surface reconstruction was $2 \times 1$ except for the special case of etching at $900 \mathrm{~K}$ where $3 \times 1$ domains were produced. LD were observed after etching at $1100 \mathrm{~K}$ and these gave rise to a weak $2 \times n$ reconstruction. Material removal was found to proceed via step retreat or layer-bylayer etching in all cases, resulting in bounded surface roughness. From the terrace etch-induced patterns on $\mathrm{Si}(100)-2 \times 1$ the energies associated with pit growth could be quantified. Such knowledge of kinetic parameters can be used for developing a comprehensive kinetic model that would allow the prediction of atomic details of surfaces exposed to various conditions of etchant flux, fluence, and temperature, and hence for the production of nanopatterned surfaces. The morphologies achieved by thermally activated reactions of adsorbed $\mathrm{F}$ with $\operatorname{Si}(100)-2 \times 1$ in the early stages reveal that dimer vacancies are also produced in the top layer but, more significantly, there is a new reaction pathway that gave rise to multilayer pitting. This pathway accounts for surface roughening which is unique to $\mathrm{F}$.

Chemisorption of $\mathrm{Br}_{2}$ on $\mathrm{Si}\left(\begin{array}{lll}1 & 1 & 1\end{array}\right)$ is dissociative, and $\mathrm{Br}$ atoms bond at adatom and restatom sites. Exposures at $500-650 \mathrm{~K}$ result in the transformation to a $\mathrm{Br}$ terminated $1 \times 1$ surface where the halogen saturates the dangling bonds and there was minimal etching. At $900 \mathrm{~K}$, the terraces are converted to $1 \times 1$ with $\mathrm{Br}$ saturation but no pitting. The steps are the active sites for etching during the initial stages of Si removal and especially following $1 \times 1$ conversion. The steps establish specific atomic structures with triangular appearance. Etching is faster at $S_{D}$ steps, and this is 
rationalized by step rebonding characteristics and the lower energy needed to remove $\mathrm{Si}$ atoms with two backbonds compared to that needed to create a kink in an $S_{N}$ step. Fast etching at steps gives rise to $\mathrm{Br}$-terminated $\mathrm{Si}_{6}$ rings as regrowth structures. Etching at $1000 \mathrm{~K}$ leads to large-scale pitting on terraces as well as step flow etching. The pits are a single bilayer deep and show characteristic $S_{N}$ bounds.

For $\operatorname{Si}(100)-2 \times 1$, terrace pits were seen for temperatures as low as $600 \mathrm{~K}$, although significant pitting was observed only for temperature at and above $800 \mathrm{~K}$. These pits were initiated as single DV and were aligned along dimer rows. Quantitative modeling of the pit shapes allowed to deduce the differences in energy barriers for etching during the dimer rows vs. branching. The steady-state morphology showed little dependence on the flux. For $\mathrm{Si}(111)-7 \times 7$ etching, terrace pitting occurs only for temperatures near $1000 \mathrm{~K}$, and pits were quite large (200-4000 А). This significant difference reflects the difference in energy associated with removal of dimer atoms from $\mathrm{Si}(100)-2 \times 1$ terraces (two back bonds) relative to terrace atoms of $\operatorname{Si}(1 \quad 11)-1 \times 1$ (three backbonds).

In this review, we have examined the progress that has been made in relating $\mathrm{Si}$ etching to the atomic scale morphologies of the surface. This spontaneous chemical etching fits in the broader context of material removal, and in that broader context we should also discuss physical removal by energetic ions (sputtering) and surface modification by photon- and electron-stimulated desorption. Such processes have been studied with STM, and the reader is referred to the literature (see, for example, [85-90] and references therein). These studies have been undertaken from the reductionist perspective - whereby a complex process (plasma-assisted material removal) is reduced to manageable model processes and the results are assembled for a broadly based understanding of the complex system. We are not yet at the assembly stage, but experiments are being designed and computational schemes are being developed that are moving us in that direction.

\section{Acknowledgements}

This work was supported by the US National Science Foundation, the US Army Research Office, and the National Council for Scientific and Technical Research (CONICET) of Argentina.

\section{References}

[1] J. Schultz, in: S.M. Sze (Ed.), VLSI Technology, McGraw-Hill, New York, 1988.

[2] H.F. Winters, J.W. Coburn, Surf. Sci. Rep. 14 (1992) 161, and references therein.

[3] G. Binnig, H. Rohrer, Helv. Phys. Acta 55 (1982) 137.

[4] R.B. Jackman, H. Ebert, J.S. Foord, Surf. Sci. 176 (1986) 183.

[5] R.B. Jackman, R.J. Price, J.S. Foord, Appl. Surf. Sci. 36 (1989) 296.

[6] Q. Gao, C.C. Cheng, P.J. Chen, W.J. Choyke, J.T. Yates Jr., J. Chem. Phys. 98 (1993) 8308.

[7] F.H.M. Sanders, A.W. Kolfshoten, J. Dieleman, R.A. Haring, A. Haring, A.E. de Vries, J. Vac. Sci. Technol. A 2 (1984) 487. 
[8] M. Chander, Y.Z. Li, J.C. Patrin, J.H. Weaver, Phys. Rev. B 47 (1993) 13035.

[9] M. Chander, Y.Z. Li, D. Rioux, J.H. Weaver, Phys. Rev. Lett. 71 (1993) 4154.

[10] D. Rioux, R.J. Pechman, M. Chander, J.H. Weaver, Phys. Rev. B 50 (1994) 4430.

[11] D. Rioux, M. Chander, Y.Z. Li, J.H. Weaver, Phys. Rev. B 49 (1994) 11071.

[12] M. Chander, D.A. Goetsch, C.M. Aldao, J.H. Weaver, Phys. Rev. Lett. 74 (1995) 2014.

[13] J.V. Seiple, J.P. Pelz, Phys. Rev. Lett. 73 (1994) 999.

[14] K.E. Johnson, T. Engel, Phys. Rev. Lett. 69 (1992) 339.

[15] K. Wurm, et al., Phys. Rev. B 50 (1994) 1567.

[16] F. Donig, A. Feltz, M. Kulakov, H.E. Hessel, U. Memmert, R.J. Behm, J. Vac. Sci. Technol. B 11 (1993) 1955.

[17] J. Seiple, J. Pecquet, Z. Meng, J.P. Pelz, J. Vac. Sci. Technol. A 11 (1993) 1649.

[18] H.E. Hessel, A. Feltz, M. Reiter, U. Memmert, R.J. Behm, Chem. Phys. Lett. 186 (1991) 275.

[19] G.J. Pietsch, U. Köhler, M. Henzler, J. Appl. Phys. 73 (1993) 4797.

[20] P. Allongue, H. Brune, H. Gerischer, Surf. Sci. 275 (1992) 414.

[21] J.J. Boland, J.S. Villarrubia, Science 248 (1990) 838.

[22] J.J. Boland, J.S. Villarrubia, Phys. Rev. B 41 (1990) 9865.

[23] J.S. Villarrubia, J.J. Boland, Phys. Rev. Lett. 63 (1989) 306.

[24] C. Yan, J.A. Jensen, A.C. Kummel, Phys. Rev. Lett. 72 (1994) 4017.

[25] R.M. Tromp, R.J. Hamers, J.E. Demuth, Phys. Rev. Lett. 55 (1985) 1303.

[26] K. Takayanagi, Y. Tanishiro, M. Takahashi, S. Takahashi, J. Vac. Sci. Technol. A 3 (1985) 1502.

[27] K.E. Johnson, T. Engel, Phys. Rev. Lett. 69 (1992) 339.

[28] C.F. Herrmann, J.J. Boland, J. Phys. Chem. B 103 (1999) 4207.

[29] C.F. Herrmann, J.J. Boland, Surf. Sci. 460 (2000) 223.

[30] I. Lyubinetsky, Z. Dohnálek, W.J. Choyke, J.T. Yates, Phys. Rev. B 58 (1998) 7950.

[31] P. Bedrossian, T. Klitsner, Phys. Rev. Lett. 68 (1992) 646.

[32] H.J.W. Zandvliet, H.B. Elswijk, E.J. van Loenen, I.S.T. Tsong, Phys. Rev. B 46 (1992) 7581.

[33] P.C. Weakliem, Z. Zhang, H. Metiu, Surf. Sci. 336 (1995) 303.

[34] H.J.W. Zandvliet, H.K. Louwsma, P.E. Hegeman, B. Poelsma, Phys. Rev. Lett. 75 (1995) 3890.

[35] M. Chander, D.A. Geotsch, C.M. Aldao, J.H. Weaver, Phys. Rev. B. 52 (1995) 8288.

[36] J.R. Sánchez, C.M. Aldao, J.H. Weaver, J. Vac. Sci. Technol. B 13 (1995) 2230.

[37] F.J. Williams, C.M. Aldao, J.H. Weaver, J. Vac. Sci. Technol. B 14 (1996) 2519.

[38] B.S. Swartzentruber, Y.-W. Mo, R. Kariotis, M.G. Lagally, M.B. Webb, Phys. Rev. Lett. 65 (1990) 1913.

[39] H.J.W. Zandvliet, H.B. Elswijk, E.J. van Loenen, D. Dijkkamp, Phys. Rev. B 45 (1992) 5965.

[40] D.J. Chadi, Phys. Rev. Lett. 39 (1987) 1691.

[41] B.S. Swartzentruber, N. Kitamura, M.G. Lagally, M.B. Webb, Phys. Rev. B 47 (1993) 13432.

[42] N. Kitamura, B.S. Swartzentruber, M.G. Lagally, M.B. Webb, Phys. Rev. B 48 (1993) 5704.

[43] H.J.W. Zandvliet, H.B. Elswijk, E.J. van Loenen, Surf. Sci. 272 (1992) 264.

[44] H.J.W. Zandvliet, H. Wormeester, D.J. Wentink, A. van Silfhout, H.B. Elswijk, Phys. Rev. Lett. 70 (1993) 2122.

[45] H.J.W. Zandvliet, B. Poelsema, H.B. Elswijk, Phys. Rev. B 51 (1995) 5465.

[46] E.D. Williams, N.C. Bartelt, Science 251 (1991) 393.

[47] N.C. Bartelt, J.L. Goldberg, T.L. Einstein, E.D. Williams, Surf. Sci. 273 (1992) 252.

[48] N.C. Bartelt, R.M. Tromp, E.D. Williams, Phys. Rev. Lett. 73 (1994) 1656.

[49] C. Pearson, B. Borovsky, M. Krueger, R. Curtis, E. Ganz, Phys. Rev. Lett. 74 (1995) 2710.

[50] F.J. Williams, J.R. Sánchez, C.M. Aldao, Surf. Sci. 391 (1997) 260.

[51] R.J. Hamers, U.K. Köhler, J.E. Demuth, Ultramicroscopy 31 (1989) 10.

[52] J.Y. Tsao, E. Chason, U. Koehler, R. Hamers, Phys. Rev. B 40 (1989) 11951.

[53] Y.-W. Mo, B. Swartzentruber, R. Kariotis, M.B. Webb, M.G. Legally, Phys. Rev. Lett. 63 (1989) 2393.

[54] Y.-W. Mo, R. Kariotis, B.S. Swartzentruber, M.B. Webb, M.G. Lagally, J. Vac. Sci. Technol. A 8 (1990) 201.

[55] G.A. de Wijs, A. De Vita, A. Selloni, Phys. Rev. Lett. 78 (1997) 4877. 
[56] K. Nakayama, C.M. Aldao, J.H. Weaver, Phys. Rev. Lett. 82 (1999) 568.

[57] K. Nakayama, C.M. Aldao, J.H. Weaver, Phys. Rev. B 59 (1999) 15893.

[58] Z. Dohnálek, H. Nishino, N. Kamoshida, J.T. Yates, J. Chem. Phys. 110 (1999) 4009.

[59] J.J. Boland, Science 262 (1993) 1703.

[60] K. Nakatsuji, K. Matsuda, T. Yonezawa, H. Daimon, S. Suga, Surf. Sci. 363 (1996) 321.

[61] D. Purdie, N.S. Prakash, K.G. Purcell, P.L. Wincott, G. Thornton, D.S.-L. Law, Phys. Rev. B 48 (1993) 2275.

[62] W.C. Simpson, J.A. Yarmoff, Surf. Sci. 359 (1996) 135.

[63] D. Rioux, F. Stepniak, R.J. Pechman, J.H. Weaver, Phys. Rev. B 51 (1995) 10981.

[64] F.J. Williams, C.M. Alado, Y. Gong, J.H. Weaver, Phys. Rev. B 55 (1997) 13829.

[65] G.A. de Wijs, A. De Vita, A. Selloni, Phys. Rev. B 57 (1998) 10021.

[66] C.G. Van de Walle, F.R. McFeely, S.T. Pantelides, Phys. Rev. Lett. 61 (1988) 1867.

[67] P.C. Weakliem, C.J. Wu, E.A. Carter, Phys. Rev. Lett. 69 (1992) 200.

[68] L.E. Carter, E.A. Carter, Surf. Sci. 323 (1995) 39.

[69] W.C. Simpson, J.A. Yarmoff, Surf. Sci. 359 (1996) 135.

[70] K.S. Nakayama, J.H. Weaver, Phys. Rev. Lett. 83 (1999) 3210.

[71] W.C. Simpson, J.A. Yarmoff, Surf. Sci. 359 (1996) 135.

[72] Y.L. Li, et al., Phys. Rev. Lett. 74 (1995) 2603.

[73] R.J. Hamers, U.K. Kohler, J. Vac. Sci. Technol. A 7 (1989) 2854.

[74] C.J. Wu, E.A. Carter, Phys. Rev. B 45 (1992) 9065.

[75] D.R. Lide (Ed.), Handbook of Chemistry and Physics, 75th Ed., CRC, Boca Raton, 1995.

[76] R.B. Jackman, H. Ebert, J.S. Foord, Surf. Sci. 176 (1989) 183.

[77] F. Owman, P. Mårtensson, Surf. Sci. 303 (1994) L367.

[78] R.J. Pechman, X.-S. Wang, J.H. Weaver, Phys. Rev. B 52 (1995) 11412.

[79] B.S. Itchkawitz, M.T. McEllistrem, J.J. Boland, Phys. Rev. Lett. 78 (1997) 98.

[80] B.S. Itchkawitz, M.T. McEllistrem, H. Grube, J.J. Boland, Surf. Sci. 385 (1997) 281.

[81] M. Fouchier, J.J. Boland, Phys. Rev. B 52 (1998) 8997.

[82] S. Ino, Jpn. J. Appl. Phys. 16 (1977) 891.

[83] R.J. Phaneuf, E.D. Williams, N.C. Bartelt, Phys. Rev. B 38 (1988) 1984.

[84] S. Kitamura, T. Sato, M. Iwatsuki, Nature 351 (1991) 215.

[85] X.-S. Wang, R.J. Pechman, J.H. Weaver, Appl. Phys. Lett. 65 (1994) 2818.

[86] R.J. Pechman, X.-S. Wang, J.H. Weaver, Phys. Rev. B 51 (1995) 10929.

[87] X.-S. Wang, R.J. Pechman, J.H. Weaver, J. Vac. Sci. Technol. B 13 (1995) 2031.

[88] X.-S. Wang, R.J. Pechman, J.H. Weaver, Surf. Sci. 364 (1996) 511.

[89] K.S. Nakayama, J.H. Weaver, Phys. Rev. Lett. 82 (1999) 980.

[90] B.Y. Han, K.S. Nakayama, J.H. Weaver, Phys. Rev. B 60 (1999) 13846. 